\title{
Khovanov homology, sutured Floer homology, and annular links
}

\author{
J. ELISENDA GRIGSBY \\ STEPHAN M. WEHRLI
}

\begin{abstract}
In [28], Lawrence Roberts, extending the work of Ozsváth and Szabó in [23], showed how to associate to a link, $\mathbb{L}$, in the complement of a fixed unknot, $B \subset S^{3}$, a spectral sequence whose $E^{2}$ term is the Khovanov homology of a link in a thickened annulus defined in [2], and whose $E^{\infty}$ term is the knot Floer homology of the preimage of $B$ inside the double-branched cover of $\mathbb{L}$.

In [6], we extended [23] in a different direction, constructing for each knot $K \subset S^{3}$ and each $n \in \mathbb{Z}_{+}$, a spectral sequence from Khovanov's categorification of the reduced, $n$-colored Jones polynomial to the sutured Floer homology of a reduced $n$-cable of $K$. In the present work, we reinterpret Roberts' result in the language of Juhász's sutured Floer homology [8] and show that the spectral sequence of [6] is a direct summand of the spectral sequence of [28].
\end{abstract}

57M27; 57R58, 81R50, 57M12

\section{Introduction}

Heegaard Floer homology [19] and Khovanov homology [10] have transformed the landscape of low-dimensional topology in the past decade, generating a wealth of applications, most notably to questions in knot concordance (cf., [16], [27], [26]), Dehn surgery (cf., [22], [29]), and contact geometry (cf., [20], [25]). The philosophies underlying the theories' constructions are quite different, yet there are intriguing connections between the two. The best-understood such connection is an algebraic relationship, discovered by Ozsváth-Szabó, between the homology of a Khovanov-type chain complex associated to a link and the homology of a Heegaard Floer-type chain complex associated to its double-branched cover.

Specifically, in [23] Ozsváth and Szabó show how to associate to a link $\mathbb{L} \subset S^{3}$ a spectral sequence whose $E^{2}$ term is $\widetilde{K h}(\overline{\mathbb{L}})$ and whose $E^{\infty}$ term is $\widehat{H F}\left(\Sigma\left(S^{3}, \mathbb{L}\right)\right)$. Here (and throughout), $\widetilde{K h}$ denotes the reduced version of Khovanov homology defined in [11], $\overline{\mathbb{L}}$ denotes the mirror of $\mathbb{L}, \Sigma(A, B)$ denotes the double-branched cover of $A$ branched over $B$, and $\widehat{H F}$ denotes (the hat version of) Heegaard Floer homology. Unless explicitly stated otherwise, all Khovanov and Heegaard Floer homology theories discussed in this paper will be considered with coefficients in $\mathbb{Z}_{2}$. 
Later work of Roberts, building on work of Plamenevskaya, demonstrated that this relationship was both useful and more general than originally believed. Specifically, given a link $\mathbb{L}$ in the complement of a fixed unknot, $B \subset S^{3}$, Roberts exhibits, in [28], the existence of a spectral sequence from $K h^{*}(\overline{\mathbb{L}})$ (where $K h^{*}$ is a version of Khovanov homology for links in product manifolds defined in [2]) to (a variant of) the knot Floer homology of $\widetilde{B} \subset \Sigma\left(S^{3}, L\right)$, where $\widetilde{B}$ is the preimage of $B$ in $\Sigma\left(S^{3}, \mathbb{L}\right)$. This allowed him to establish a relationship, first conjectured in [25], between Plamenevskaya's transverse invariant [25] and Ozsváth-Szabó's contact invariant [20]. Baldwin and Plamenevskaya, in [4], used (an extension of) this relationship to establish the tightness of a number of non Stein-fillable contact structures.

In [6], we extended [23] in a different direction, using a version of Heegaard Floer homology for sutured manifolds (Definition 2.3) developed by Juhász in [8], yielding a proof that a simple variant of Khovanov homology, categorifying the reduced, $n$-colored Jones polynomial and defined in [12], detects the unknot whenever $n \geq 2$.

The goal of the present work is to propose a single general framework unifying all of these results. As in [6], this general framework uses Gabai's sutured manifold theory [5] and Juhász's sutured Floer homology [8]. It not only incorporates all known results of this type but also clarifies their relationship to each other. In particular, it can be shown to satisfy nice naturality properties with respect to certain TQFT operations, as detailed in [7].

More specifically, let $F$ be an oriented surface with $\partial F \neq \emptyset, F \times I$ a product sutured manifold (see Definition 2.3 and Example 2.4), and $T \subset F \times I$ a tangle (properly imbedded 1 -manifold) that is both admissible, i.e.,

$$
T \cap(\partial F \times I)=\emptyset
$$

and balanced, i.e.,

$$
|T \cap(F \times\{1\})|=|T \cap(F \times\{0\})|=n \in \mathbb{Z}_{\geq 0} .
$$

Then there should exist a spectral sequence whose $E^{2}$ term is an appropriate version of the Khovanov homology of $\bar{T}$, which we will denote $K h^{*}(\bar{T})$, and whose $E^{\infty}$ term is the sutured Floer homology of $\Sigma(F \times I, T)$, which we will denote $\operatorname{SFH}(\Sigma(F \times I, T))$. We shall explore these ideas more fully in the sequel-in particular, the appropriate version of Khovanov homology for balanced tangles in product sutured manifolds should be similar to what appears in [1], with abelianized gradings-focusing here on the case when $F=A$ is an annulus and $T=\mathbb{L}$ is a 0 -balanced tangle (link):

Theorem 2.1 Let $\mathbb{L} \subset A \times I$ be a link in the product sutured manifold $A \times I$. Then there is a spectral sequence whose $E^{2}$ term is $K h^{*}(\overline{\mathbb{L}})$ and whose $E^{\infty}$ term is $\operatorname{SFH}(\Sigma(A \times I, \mathbb{L}))$. 
Note that we treated the case when $F=D$ in [6]:

Theorem 1.1 [6, Prop. 5.20] Let $T \subset D \times I$ be an admissible, balanced tangle. Then there is a spectral sequence whose $E^{2}$ term is $K^{*}(\bar{T})$ and whose $E^{\infty}$ term is $\operatorname{SFH}(\Sigma(D \times I, T)) .{ }^{1}$

In fact, Theorem 2.1 is a reinterpretation (and modest extension) of Roberts' main result in [28]. (Note that in [28], Roberts restricts to those $\mathbb{L}$ intersecting a spanning disk of $B$ in an odd number of points, while Theorem 2.1 requires no such restriction.) To understand the connection, let $A$ be an oriented annulus, $I=[0,1]$ the oriented closed unit interval, and $\mathbb{L} \subset A \times I$ a link, where $A \times I$ has been identified as the standard sutured complement of a standardly-imbedded unknot, $B \subset S^{3}$, via the identification:

$$
\begin{aligned}
A \times I & =\{(r, \theta, z) \mid r \in[1,2], \theta \in[0,2 \pi), z \in[0,1]\} \subset \mathbb{R}^{3} \cup \infty=S^{3}, \\
B & =\{(r, \theta, z) \mid r=0\} \cup \infty \subset S^{3} .
\end{aligned}
$$

In [28, Prop. 1.1], Roberts constructs a spectral sequence from $K h^{*}(\overline{\mathbb{L}})$, where $K h^{*}$ is a version of Khovanov homology for links in product manifolds defined in [2] ${ }^{2}$ to (a variant of) the knot Floer homology of $\widetilde{B} \subset \Sigma\left(S^{3}, \mathbb{L}\right)$, where $\widetilde{B}$ is the preimage of $B$ in $\Sigma\left(S^{3}, \mathbb{L}\right)$. We show (see Proposition 2.24) that this (variant of) the knot Floer homology of $\widetilde{B}$ is, in fact, just the sutured Floer homology of $\Sigma(A \times I, \mathbb{L})$.

Furthermore, there is a nice relationship between the spectral sequences of Theorems 1.1 and 2.1. Specifically a link $\mathbb{L} \subset A \times I$ can be cut along a vertical disk as in Figure 1 to form an admissible, balanced tangle $T \subset D \times I$. The following is a corollary of [7, Thm. 4.5].

Theorem 3.1 Let $\mathbb{L} \subset A \times I$ be an isotopy class representative of an annular link admitting a projection, $\mathcal{P}(\mathbb{L})$, and let $\lambda \subset A$ be a properly imbedded oriented arc representing a nontrivial element of $H_{1}(A, \partial A)$ such that $\lambda$ intersects $\mathcal{P}(\mathbb{L})$ transversely. Let $T \subset D \times I$ be the balanced tangle in $D \times I$ obtained by decomposing $A \times I$ (Definition 2.8) along the surface $\lambda \times I$ endowed with the product orientation.

Then the spectral sequence

$$
K h^{*}(\bar{T}) \rightarrow \operatorname{SFH}(\Sigma(D \times I, T))
$$

is a direct summand of the spectral sequence

$$
K h^{*}(\overline{\mathbb{L}}) \rightarrow \operatorname{SFH}(\Sigma(A \times I, \mathbb{L})) .
$$

Furthermore, the direct summand is trivial if there exists some $\mathbb{L}^{\prime} \subset A \times I$ isotopic to $\mathbb{L}$ satisfying

$$
\left|(\lambda \times I) \pitchfork \mathbb{L}^{\prime}\right| \lesseqgtr|(\lambda \times I) \pitchfork \mathbb{L}|
$$




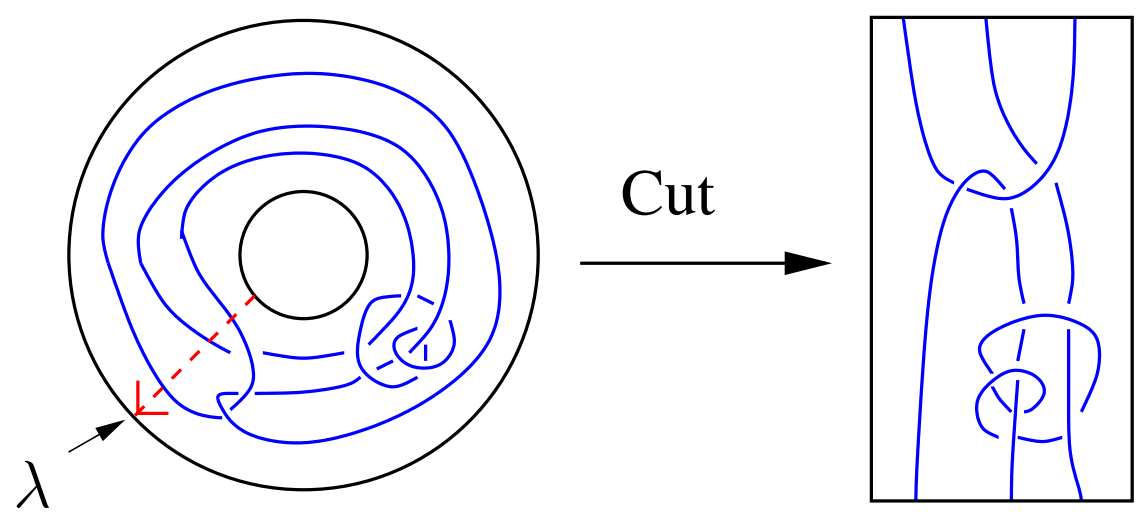

Figure 1: Cutting an annular link projection along a homologically non-trivial oriented arc

Theorem 3.1 can be viewed as the first example of the "naturality" of the relationship between Khovanov homology and Heegaard Floer homology; under various natural geometric operations, the spectral sequence behaves "as expected." See [7] for more examples.

It is also interesting to note that, given a link, $\mathbb{L} \subset S^{3}$, any unknot, $B \subset S^{3}-N(\mathbb{L})$, endows the Khovanov chain complex associated to $\mathbb{L} \subset B^{3}$ with a $\mathbb{Z}$-filtration, via the identification

$$
S^{3}-N(B) \leftrightarrow A \times I
$$

(see [28] and Proposition 2.24). The extra grading inducing this $\mathbb{Z}$-filtration has the following representation-theoretic interpretation. Suppose $T \subset D \times I$ is an $n$-balanced tangle obtained by decomposing $\mathbb{L} \subset A \times I$ along $\lambda \times I$, as in Theorem 3.1. Then the $U_{q}\left(\mathfrak{s} l_{2}\right)$ tangle invariant associated to $T$ is an endomorphism, $J(T)$, of the $U_{q}\left(\mathfrak{s} l_{2}\right)$-module $V^{\otimes n}$, where $V$ denotes the standard 2-dimensional irreducible representation of $U_{q}\left(\mathfrak{s l} l_{2}\right)$. Denoting by $a_{k}(T) \in \mathbb{Z}\left[q^{ \pm 1}\right]$ the trace of the restriction of $J(T)$ to the $k$-th weight space in $V^{\otimes n}$, one can show that the sum,

$$
\sum_{k \in Z}(t q)^{k} a_{k}(T) \in \mathbb{Z}\left[q^{ \pm 1}, t^{ \pm 1}\right]
$$

is precisely the graded Euler characteristic, $\left.\chi_{t, q}\left(K h^{*}(\mathbb{L})\right)\right)$, of $K h^{*}(\mathbb{L})$, defined as in [28], with the power of $t$ corresponding to the extra grading. Thus, the extra grading is related to the decomposition of $J(T)$ according to weight spaces in $V^{\otimes n}$.

We also remark that choosing an alternative rational slope on $\partial\left(S^{3}-N(B)\right)$ corresponds to choosing an alternative Dehn filling of $B$, furnishing a plausible means of constructing Khovanov-type invariants for links in lens spaces.

${ }^{1} K h^{*}(\bar{T})$ is denoted $V(T)$ in [6, Sec. 5].

${ }^{2} K h^{*}(\mathbb{L})$ is denoted $H(\mathbb{L})$ in [28, Sec. 2]. 
Acknowledgments: This work has benefited greatly from conversations with Ken Baker, John Baldwin, Matt Hedden, András Juhász, Mikhail Khovanov, Rob Kirby, Robert Lipshitz, Peter Ozsváth, Lawrence Roberts, and Liam Watson. In addition, a large portion of this work was completed while both authors were visiting postdoctoral fellows at Columbia University. We are grateful to the Columbia mathematics department for its hospitality. The first author was partially supported by an NSF postdoctoral fellowship and NSF grant number DMS-0905848. The second author was partially supported by a Swiss NSF fellowship for prospective researchers and a postdoctoral fellowship of the Fondation Sciences Mathématiques de Paris.

\section{Spectral Sequence from Khovanov to Sutured Floer}

This section is devoted to proving the main theorem:

Theorem 2.1 Let $\mathbb{L} \subset A \times I$ be a link in the product sutured manifold $A \times I$. Then there exists a spectral sequence from $K h^{*}(\overline{\mathbb{L}})$ to $\operatorname{SFH}(\Sigma(A \times I, \mathbb{L}))$.

In the above, $K h^{*}(\overline{\mathbb{L}})$ is the homology of a Khovanov-type chain complex associated to $\overline{\mathbb{L}}$, the mirror of $\mathbb{L}$, while $S F H(\Sigma(A \times I, \mathbb{L}))$ is the sutured Floer homology of $\Sigma(A \times I, \mathbb{L})$, the homology of a Heegaard Floer-type chain complex. Both constructions begin by associating a cube of resolutions to a projection (diagram) of $\mathbb{L} \subset A \times I$. As in [6], the main step in the proof will be a verification of the equivalence of Khovanov and sutured Floer "functors" applied to the vertices and edges of this cube. We begin by fixing notation and recalling some standard definitions in Section 2.1. We go on to describe the Khovanov and sutured Floer functors on resolved link projections in Sections 2.2 and 2.3, establishing their equivalence in Proposition 2.29.

\subsection{Notation and Standard Definitions}

Notation 2.2 Throughout, $A$ denotes an oriented annulus and $I:=[0,1]$ denotes the oriented closed unit interval.

Whenever we write $A \times I$, we shall always assume we have fixed an identification:

$$
\left.A \times I:=\{(r, \theta, z) \mid r \in[1,2], z \in[0,1]\} \subset \mathbb{R}^{2} \times \mathbb{R} .\right\}
$$

Let $A_{+}$(resp., $A_{-}$) denote $A \times\{1\}$ (resp., $A \times\{0\}$ ). 
Most of the following definitions can be found in [5], [8], [9], and [6]. See also [10] and [19].

Definition 2.3 [5] A sutured manifold $(Y, \Gamma)$ is a compact, oriented 3-manifold with boundary $\partial Y$ along with a set $\Gamma \subset \partial Y$ of pairwise disjoint annuli $A(\Gamma)$ and tori $T(\Gamma)$. The interior of each component of $A(\Gamma)$ contains a suture, an oriented simple closed curve which is homologically nontrivial in $A(\Gamma)$. The union of the sutures is denoted $s(\Gamma)$.

Every component of $R(\Gamma)=\partial Y-\operatorname{Int}(\Gamma)$ is assigned an orientation compatible with the oriented sutures. More precisely, if $\delta$ is a component of $\partial R(\Gamma)$, endowed with the boundary orientation, then $\delta$ must represent the same homology class in $H_{1}(\Gamma)$ as some suture. Let $R_{+}(\Gamma)$ (resp., $R_{-}(\Gamma)$ ) denote those components of $R(\Gamma)$ whose normal vectors point out of (resp., into) $Y$.

We omit mention of the distinguished annuli, $\Gamma$, from the notation when $Y$ is one of the following standard sutured manifolds:

Example 2.4 Let $F$ be an oriented surface with $\partial F \neq \emptyset$. Then $F \times I$ denotes the product sutured manifold $(F \times I, \Gamma)$, where $\Gamma=\partial F \times I$, and $s(\Gamma)=\partial F \times\left\{\frac{1}{2}\right\}$.

Example 2.5 Let $(Y, \Gamma)$ be a sutured manifold and

$$
(B, \partial B) \subset(Y, \partial Y)
$$

a smoothly imbedded 1-submanifold satisfying

$$
\partial B \cap \Gamma=\emptyset .
$$

Let $\Sigma(Y, B)$ be any two-fold cyclic branched cover of $Y$ over $B$ with covering map

$$
\pi: \Sigma(Y, B) \rightarrow Y
$$

Then we denote by $(\Sigma(Y, B), \widetilde{\Gamma})$ the sutured manifold with $\widetilde{\Gamma}=\pi^{-1}(\Gamma)$ and sutures $s(\widetilde{\Gamma})=\pi^{-1}(s(\Gamma))$. If $(Y, \Gamma)$ is a product sutured manifold, then we omit mention of $\widetilde{\Gamma}$ from the notation for $\Sigma(Y, B)$.

Remark 2.6 Note that in general the construction of a 2 -fold cyclic branched cover of $Y$ over $B$ depends upon a choice of homomorphism

$$
\phi: H_{1}(Y-B ; \mathbb{Z}) \rightarrow \mathbb{Z}_{2}
$$

In the present work, we make the following canonical choices: 
(1) When $Y=D \times I$ and $B=T \subset D \times I$ is a balanced tangle with $m$ connected components,

$$
H_{1}(Y-B ; \mathbb{Z}) \cong \mathbb{Z}^{m}
$$

with basis given by the meridians $\mu_{1}, \ldots, \mu_{m}$. We choose the homomorphism, $\phi$, satisfying $\phi\left(\mu_{i}\right)=1 \in \mathbb{Z}_{2}$ for all $\mu_{i}$.

(2) When $Y=A \times I$ and $B=\mathbb{L} \subset A \times I$ is a 0 -balanced tangle (link) with $m$ connected components,

$$
H_{1}(Y-B ; \mathbb{Z}) \cong \mathbb{Z}^{m+1}
$$

with basis given by the meridians $\mu_{1}, \ldots, \mu_{m}$ along with $\gamma$, where $\gamma$ is represented by either connected component of $s(\Gamma)$. We choose the homomorphism, $\phi$, satisfying $\phi\left(\mu_{i}\right)=1$ for all $\mu_{i}$ and $\phi(\gamma)=0$. Note that, since the two components of $s(\Gamma)$ are homologous mod 2 in $Y-B$, the choice of $\gamma$ is irrelevant.

Definition 2.7 [9, Defn. 2.7] A decomposing surface in a sutured manifold, $(Y, \Gamma)$, is a properly imbedded, oriented surface, $(S, \partial S) \subset(Y, \partial Y)$ such that for every component, $\lambda$, of $(\partial S) \cap \Gamma$, one of the following occurs:

- $\lambda$ is a properly imbedded non-separating $\operatorname{arc}$ in $\Gamma$ such that $|\lambda \cap s(\Gamma)|=1$.

- $\lambda$ is a simple closed curve in an annular component, $A$, of $\Gamma$ representing the same homology class in $A$ as $s(\Gamma)$.

- $\lambda$ is a homotopically nontrivial curve in a torus component $T$ of $\Gamma$, and if $\delta$ is another component of $T \cap(\partial S)$, then $\lambda$ and $\delta$ represent the same homology class in $T$.

Definition 2.8 Given a decomposing surface, $S$, in a sutured manifold, $Y$, the result of decomposing $Y$ along $S$ is a new sutured manifold, $\left(Y^{\prime}, \Gamma^{\prime}\right)$, obtained as follows.

- $Y^{\prime}=Y-\operatorname{Int}(N(S))$,

- $\Gamma^{\prime}=\left(\Gamma \cap Y^{\prime}\right) \cup N\left(S_{+}^{\prime} \cap R_{-}(\Gamma)\right) \cup N\left(S_{-}^{\prime} \cap R_{+}(\Gamma)\right)$,

- $R_{+}\left(\Gamma^{\prime}\right)=\left(\left(R_{+}(\Gamma) \cap Y^{\prime}\right) \cup S_{+}^{\prime}\right)-\operatorname{Int}\left(\Gamma^{\prime}\right)$,

- $R_{-}\left(\Gamma^{\prime}\right)=\left(\left(R_{-}(\Gamma) \cap Y^{\prime}\right) \cup S_{-}^{\prime}\right)-\operatorname{Int}\left(\Gamma^{\prime}\right)$,

where $S_{+}^{\prime}$ (resp., $S_{-}^{\prime}$ ) is the component of $\partial N(S) \cap Y^{\prime}$ whose normal vector field points out of (resp., into) $Y^{\prime}$.

Definition 2.9 A link $\mathbb{L} \subset A \times I$ is a smoothly imbedded, unoriented, closed 1-manifold. Two such imbeddings $\mathbb{L}_{1}, \mathbb{L}_{2}$ are said to be equivalent if there is an ambient isotopy taking $\mathbb{L}_{1}$ to $\mathbb{L}_{2}$ which acts trivially on $\partial A \times I$. A knot is a 1 -component link. 
Note that we require our ambient isotopy to act trivially on $\Gamma=\partial A \times I$, the neighborhood of the sutures, in order to preserve the structure of the ambient manifold, $A \times I$ as a sutured manifold.

Definition 2.10 Let $\pi_{z}: A \times I \rightarrow A$ be the standard projection defined by

$$
\pi_{z}(r, \theta, z)=(r, \theta)
$$

For any link $\mathbb{L} \subset A \times I$ for which $\pi_{z}(\mathbb{L}) \subset A$ is a smooth imbedding away from finitely many transverse double points, we denote by $\mathcal{P}(\mathbb{L})$ the enhancement of $\pi_{z}(\mathbb{L})$ which encodes over/undercrossing information. We call $\mathcal{P}(\mathbb{L})$ the projection of $\mathbb{L}$.

Definition 2.11 A link $\mathbb{L} \subset A \times I$ is said to be resolved if $\mathcal{P}(\mathbb{L})$ is an imbedding.

Definition 2.12 A saddle cobordism $S \subset A \times[0,1]$ is a smooth cobordism between two resolved link projections $\mathcal{P}\left(\mathbb{L}^{\prime}\right)$ and $\mathcal{P}\left(\mathbb{L}^{\prime \prime}\right)$ with the property that $\exists$ a unique $c \in[0,1]$ such that

(1) $S \cap(A \times\{c\})$ is a smooth 1-dimensional imbedding away from a single double-point.

(2) $S \cap(A \times\{s\})$ is a smooth 1 -dimensional imbedding whenever $s \neq c$.

Let $\left|\mathbb{L}^{\prime}\right|$ (resp., $\left|\mathbb{L}^{\prime \prime}\right|$ ) denote the number of connected components of $\mathbb{L}^{\prime}$ (resp., $\mathbb{L}^{\prime \prime}$ ). There are two cases:

(1) When $\left|\mathbb{L}^{\prime}\right|=\left|\mathbb{L}^{\prime \prime}\right|+1$, we call $S$ a merge saddle cobordism, and

(2) when $\left|\mathbb{L}^{\prime}\right|=\left|\mathbb{L}^{\prime \prime}\right|-1$, we call $S$ a split saddle cobordism.

\subsection{Khovanov functor}

Let $\mathbb{L} \subset A \times I$ be a resolved link with connected components $K_{1}, \ldots, K_{t}, K_{t+1}, \ldots, K_{t+n}$, where

$$
\mathcal{P}\left(K_{i}\right) \quad \begin{cases}=0 \in H_{1}(A ; \mathbb{Z}) & \text { if } i \in\{1, \ldots, t\}, \text { and } \\ \neq 0 \in H_{1}(A ; \mathbb{Z}) & \text { if } i \in\{t+1, \ldots, t+n\}\end{cases}
$$

Denote

$$
\begin{aligned}
\mathbb{L}_{\text {triv }} & :=K_{1} \amalg \ldots \amalg K_{t} \\
\mathbb{L}_{n o n} & :=K_{t+1} \amalg \ldots \amalg K_{t+n} .
\end{aligned}
$$


Definition 2.13 For $\mathbb{L}=K_{1} \amalg \ldots \amalg K_{t+n} \subset A \times I$ a resolved link, let $Z(\mathbb{L})$ denote the $\mathbb{Z}_{2}$-vector space formally generated by $\left[K_{1}\right], \ldots,\left[K_{t+n}\right]$ :

$$
Z(\mathbb{L}):=\operatorname{Span}_{\mathbb{Z}_{2}}\left(\left[K_{1}\right], \ldots,\left[K_{t+n}\right]\right) .
$$

Define a bigrading on $Z(\mathbb{L})$ by

$$
\operatorname{deg}\left(\left[K_{i}\right]\right):= \begin{cases}(0,-2) & \text { for } 1 \leq i \leq t, \text { and } \\ (-2,-2) & \text { else. }\end{cases}
$$

The space $Z(\mathbb{L})$ decomposes as $Z(\mathbb{L})=Z\left(\mathbb{L}_{\text {triv }}\right) \oplus Z\left(\mathbb{L}_{\text {non }}\right)$, where

$$
\begin{aligned}
Z\left(\mathbb{L}_{\text {triv }}\right) & :=\operatorname{Span}_{\mathbb{Z}_{2}}\left(\left[K_{1}\right], \ldots,\left[K_{t}\right]\right) . \\
Z\left(\mathbb{L}_{\text {non }}\right) & :=\operatorname{Span}_{\mathbb{Z}_{2}}\left(\left[K_{t+1}\right], \ldots,\left[K_{t+n}\right]\right) .
\end{aligned}
$$

Definition 2.14 Let $\bar{V}(\mathbb{L})$ denote the exterior algebra

$$
\bar{V}(\mathbb{L}):=\Lambda^{*} Z(\mathbb{L}),
$$

i.e. the polynomial algebra over $\mathbb{Z}_{2}$ in formal variables $\left[K_{1}\right], \ldots,\left[K_{t+n}\right]$ satisfying the relations $\left[K_{1}\right]^{2} \sim \ldots \sim\left[K_{t+n}\right]^{2} \sim 0$. The bigrading on $Z(\mathbb{L})$ induces a bigrading on $\bar{V}(\mathbb{L})$, via

$$
\operatorname{deg}\left(\left[K_{i_{1}}\right] \wedge \ldots \wedge\left[K_{i_{k}}\right]\right):=\operatorname{deg}\left(\left[K_{i_{1}}\right]\right)+\ldots+\operatorname{deg}\left(\left[K_{i_{k}}\right]\right) .
$$

Given a bigraded vector space $V$ and a pair $(a, b) \in \mathbb{Z}^{2}$, we denote by $V\{a, b\}$ the vector space $V$, with gradings shifted by $(a, b)$. I.e., $\operatorname{deg}_{V\{a, b\}}(v):=\operatorname{deg}_{V}(v)+(a, b)$ for all $v \in V$. Let

$$
V(\mathbb{L}):=\bar{V}(\mathbb{L})\{n, t+n\} .
$$

We can write $V(\mathbb{L})$ as a direct sum

$$
V(\mathbb{L})=\bigoplus_{f, q \in \mathbb{Z}} V(\mathbb{L} ; f, q)
$$

where $V(\mathbb{L} ; f, q)$ denotes the subspace consisting of all $v \in V(\mathbb{L})$ with $\operatorname{deg}(v)=(f, q)$.

Now consider a merge saddle cobordism $S_{m} \subset A \times[0,1]$ between two resolved link projections $\mathcal{P}\left(\mathbb{L}^{\prime}\right)$ and $\mathcal{P}\left(\mathbb{L}^{\prime \prime}\right)$, where the saddle merges two components of $\mathbb{L}^{\prime}$ labeled $K_{i}^{\prime}$ and $K_{j}^{\prime}$. Then there is a natural identification

$$
Z\left(\mathbb{L}^{\prime}\right) /\left[K_{i}^{\prime}\right] \sim\left[K_{j}^{\prime}\right]=Z\left(\mathbb{L}^{\prime \prime}\right)
$$

and, correspondingly, there is an isomorphism

$$
\alpha: V\left(\mathbb{L}^{\prime}\right) /\left[K_{i}^{\prime}\right] \sim\left[K_{j}^{\prime}\right] \stackrel{\cong}{\longrightarrow} V\left(\mathbb{L}^{\prime \prime}\right) .
$$


Definition 2.15 Associated to $S_{m}$ is a linear map $\mathcal{V}_{m}: V\left(\mathbb{L}^{\prime}\right) \rightarrow V\left(\mathbb{L}^{\prime \prime}\right)$, referred to as the multiplication map, and defined as the composite

$$
V\left(\mathbb{L}^{\prime}\right) \stackrel{\pi}{\longrightarrow} \frac{V\left(\mathbb{L}^{\prime}\right)}{\left[K_{i}^{\prime}\right] \sim\left[K_{j}^{\prime}\right]} \stackrel{\alpha}{\longrightarrow} V\left(\mathbb{L}^{\prime \prime}\right),
$$

where $\pi$ denotes the quotient map. Let $G \mathcal{V}_{m}$ denote the part of $\mathcal{V}_{m}$ which is $f$-grading preserving on $V\left(\mathbb{L}^{\prime}\right), V\left(\mathbb{L}^{\prime \prime}\right)$. More explicitly, if $\mathbf{x} \in V\left(\mathbb{L}^{\prime \prime}\right)$ is a homogeneous element in $f$-grading $f_{0}$, then

$$
G \mathcal{V}_{m}(\mathbf{x}):=p_{f_{0}} \circ \mathcal{V}_{m}(\mathbf{x}),
$$

where $p_{f_{0}}$ is the projection map $p_{f_{0}}: V\left(\mathbb{L}^{\prime \prime}\right) \rightarrow V\left(\mathbb{L}^{\prime \prime} ; f=f_{0}\right)$. Now extend linearly.

Running the merge saddle cobordism $S_{m}$ backwards produces a split saddle cobordism $S_{\Delta}: \mathcal{P}\left(\mathbb{L}^{\prime \prime}\right) \rightarrow \mathcal{P}\left(\mathbb{L}^{\prime}\right)$.

Definition 2.16 Associated to $S_{\Delta}$ is a comultiplication map $\mathcal{V}_{\Delta}: V\left(\mathbb{L}^{\prime \prime}\right) \rightarrow V\left(\mathbb{L}^{\prime}\right)$, defined as the composite

$$
V\left(\mathbb{L}^{\prime \prime}\right) \stackrel{\alpha^{-1}}{\longrightarrow} \frac{V\left(\mathbb{L}^{\prime}\right)}{\left[K_{i}^{\prime}\right] \sim\left[K_{j}^{\prime}\right]} \stackrel{\varphi}{\longrightarrow} V\left(\mathbb{L}^{\prime}\right),
$$

with $\varphi$ given by $\varphi(a):=\left(\left[K_{i}^{\prime}\right]+\left[K_{j}^{\prime}\right]\right) \wedge \widetilde{a}$, where $\widetilde{a}$ is any lift of $a$ in $\pi^{-1}(a)$. As above, we denote by $G \mathcal{V}_{\Delta}$ the part of $\mathcal{V}_{\Delta}$ which is $f$-grading perserving, i.e., $G \mathcal{V}_{\Delta}$ linearly extends the map which sends elements $\mathbf{x} \in V\left(\mathbb{L}^{\prime \prime}\right)$ in $f$-grading $f_{0}$ to

$$
p_{f_{0}} \circ \mathcal{V}_{\Delta}(\mathbf{x}) \text {. }
$$

The maps $G \mathcal{V}_{m}, G \mathcal{V}_{\Delta}$ are used to define chain complexes associated to a projection $\mathcal{P}(\mathbb{L})$ of a link $\mathbb{L} \subset A \times I$ as follows.

Label the crossings of $\mathcal{P}(\mathbb{L})$ by $1, \ldots, \ell$. For any $\ell$-tuple $\mathcal{I}=\left(m_{1}, \ldots, m_{\ell}\right) \in\{0,1, \infty\}^{\ell}$, we denote by $\mathcal{P}_{\mathcal{I}}(\mathbb{L})$ the link projection obtained from $\mathcal{P}(\mathbb{L})$ by

- leaving a neighborhood of the $i$ th crossing unchanged, if $m_{i}=\infty$,

- replacing a neighborhood of the $i$ th crossing with a " 0 " resolution, if $m_{i}=0$, and

- replacing a neighborhood of the $i$ th crossing with a "1" resolution, if $m_{i}=1$.

See Figure 2. Note that our conventions for the "0" and "1" resolutions of a crossing match those of [23] and [6], which are opposite of Khovanov's standard convention [10], used by Roberts in [28].

Definition 2.17 Giving the set $\{0,1, \infty\}$ the dictionary ordering, we call an $\ell$-tuple $\mathcal{I}^{\prime} \in\{0,1, \infty\}^{\ell}=\left(m_{1}, \ldots, m_{\ell}\right)$ an immediate successor of $\mathcal{I}$ if there exists some $j$ such that $m_{i}=m_{i}^{\prime}$ if $i \neq j$ and $\left(m_{j}, m_{j}^{\prime}\right)$ is either $(0,1)$ or $(1, \infty)$. 


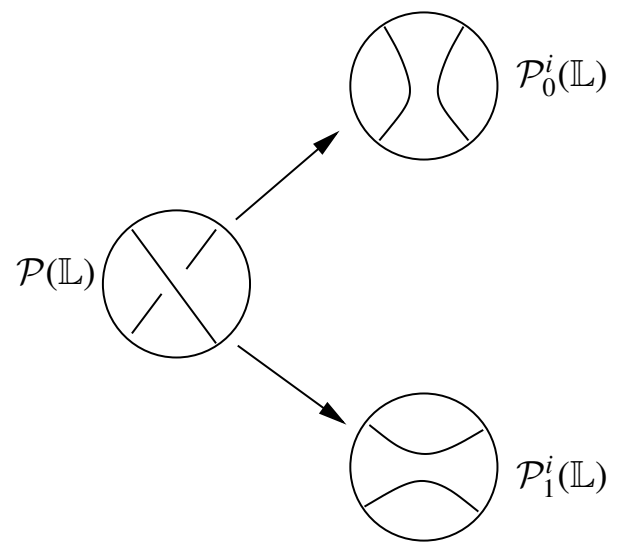

Figure 2: Replacing the $i$-th crossing of a projection, $\mathcal{P}(\mathbb{L})$, with a 0 or 1 resolution

Definition 2.18 Given a projection $\mathcal{P}(\mathbb{L}) \subset A$ of a link $\mathbb{L} \subset A \times I$, we define a chain complex

$$
C V(\mathcal{P}(\mathbb{L}))=\left(\bigoplus_{\mathcal{I} \in\{0,1\}^{\ell}} V\left(P_{\mathcal{I}}(\mathbb{L})\right), D\right),
$$

where $D=\sum_{\mathcal{I}, \mathcal{I}^{\prime}} D_{\mathcal{I}, \mathcal{I}^{\prime}}$, with the sum taken over all pairs $\mathcal{I}, \mathcal{I}^{\prime} \in\{0,1\}^{\ell}$ such that $\mathcal{I}^{\prime}$ is an immediate successor of $\mathcal{I}$, and

$$
D_{\mathcal{I}, \mathcal{I}^{\prime}}: V\left(\mathcal{P}_{\mathcal{I}}(\mathbb{L})\right) \rightarrow V\left(\mathcal{P}_{\mathcal{I}^{\prime}}(\mathbb{L})\right)
$$

is given by $\mathcal{V}_{m}$ (resp., $\mathcal{V}_{\Delta}$ ) when $\mathcal{P}_{\mathcal{I}^{\prime}}(\mathbb{L})$ is obtained from $\mathcal{P}_{\mathcal{I}}(\mathbb{L})$ by a merge (resp., split) saddle cobordism.

If $\mathbb{L}$ is equipped with an orientation, then the complex $C V(\mathcal{P}(\mathbb{L}))$ can be endowed with three $\mathbb{Z}$ gradings, called the $i, j$ and $k$-grading.

Definition 2.19 An element $v \in C V(\mathcal{P}(\mathbb{L}))$ is said to have degree $\operatorname{deg}(v)=(i, j, k) \in \mathbb{Z}^{3}$ if it is contained in the subspace

$$
C V(\mathcal{P}(\mathbb{L}) ; i, j, k):=\bigoplus V_{\mathcal{I}}(\mathcal{P}(\mathbb{L}) ; f, q) \subset C V(\mathcal{P}(\mathbb{L}))
$$

where the sum ranges over all triples $(\mathcal{I}, q, f) \in\{0,1\}^{\ell} \times \mathbb{Z}^{2}$ satisfying

$$
\begin{aligned}
i & =|\mathcal{I}|-n_{+}, \\
j & =q+|\mathcal{I}|+n_{-}-2 n_{+}, \\
k & =f
\end{aligned}
$$


where $n_{+}$(resp., $n_{-}$) denotes the number of positive (resp., negative) crossings in $\mathcal{P}(\mathbb{L}$ ), and $|\mathcal{I}|$ denotes the number of 1 's in the $\ell$-tuple $\mathcal{I} \in\{0,1\}^{\ell}$.

Remark 2.20 For a resolved link, $\mathbb{L} \subset A \times I$, our $V(\mathbb{L})$ is equivalent, as a $\mathbb{Z}_{2}$ vector space, to what Roberts denotes $V(\mathbb{L})$ in [28, Sec. 2], and our $(f, q)$-bigrading matches his for resolved links. For an unresolved link $\mathbb{L}$, Roberts' $f$-grading (for $\overline{\mathbb{L}}$ ) matches ours (for $\mathbb{L}$ ), and his $q$-grading matches our $j$-grading (which in turn matches our $q$-grading up to an overall shift). Note also that, if one forgets the $k$-grading, then $C V(\mathcal{P}(\mathbb{L}))$ becomes the chain complex that Ozsváth and Szabó associate to $\mathbb{L} \subset S^{3}$ in [23]. In particular, the homology of $C V(\mathcal{P}(\mathbb{L}))$ is the Khovanov homology of $\overline{\mathbb{L}} \subset S^{3}$.

Lemma 2.21 The differential, $D$, on $C V(\mathcal{P}(\mathbb{L}))$, is non-increasing in the $k$ grading. Hence, $C V(\mathcal{P}(\mathbb{L}))$ has the structure of a $\mathbb{Z}$-filtered chain complex, with the $\mathbb{Z}$-filtration induced by the $k$-grading.

Proof Let $\mathcal{I}, \mathcal{I}^{\prime} \in\{0,1\}^{\ell}$, where $\mathcal{I}^{\prime}$ is an immediate successor of $\mathcal{I}$, and let $\mathcal{P}_{\mathcal{I}}(\mathbb{L}), \mathcal{P}_{\mathcal{I}^{\prime}}(\mathbb{L})$ denote the associated resolutions.

Suppose that $\mathcal{P}_{\mathcal{I}^{\prime}}(\mathbb{L})$ is obtained from $\mathcal{P}_{\mathcal{I}}(\mathbb{L})$ by merging two components, $K_{i}$ and $K_{j}$, of $\mathcal{P}_{\mathcal{I}}(\mathbb{L})$ to form a component, $K^{\prime}$, of $\mathcal{P}_{\mathcal{I}^{\prime}}(\mathbb{L})$. Let $\operatorname{deg}_{f}\left(K_{i}\right), \operatorname{deg}_{f}\left(K_{j}\right)$ (resp., $\left.\operatorname{deg}_{f}\left(K^{\prime}\right)\right)$ denote their $f$-degrees as elements of $Z\left(\mathcal{P}_{\mathcal{I}}(\mathbb{L})\right)$ (resp., $Z\left(\mathcal{P}_{\mathcal{I}^{\prime}}(\mathbb{L})\right)$ ).

There are three possibilities:

(1) $K_{i}, K_{j} \subset\left(\mathcal{P}_{\mathcal{I}}(\mathbb{L})\right)_{\text {triv }}$

(2) one of $K_{i}, K_{j}$ is in $\left(\mathcal{P}_{\mathcal{I}}(\mathbb{L})\right)_{\text {triv }}$, while the other is in $\left(\mathcal{P}_{\mathcal{I}}(\mathbb{L})\right)_{\text {non }}$, or

(3) $K_{i}, K_{j} \subset\left(\mathcal{P}_{\mathcal{I}}(\mathbb{L})\right)_{\text {non }}$.

In the first two cases,

$$
\operatorname{deg}_{f}\left(K^{\prime}\right)=\min \left\{\operatorname{deg}_{f}\left(K_{i}\right), \operatorname{deg}_{f}\left(K_{j}\right)\right\},
$$

and since, in these two cases, $\mathcal{P}_{\mathcal{I}}(\mathbb{L})$ and $\mathcal{P}_{\mathcal{I}^{\prime}}(\mathbb{L})$ have the same number of non-trivial components, the $f$-grading shift relating $V\left(\mathcal{P}_{\mathcal{I}}(\mathbb{L})\right)$ to $\bar{V}\left(\mathcal{P}_{\mathcal{I}}(\mathbb{L})\right)$ is the same as the $f$ grading shift relating $V\left(\mathcal{P}_{\mathcal{I}^{\prime}}(\mathbb{L})\right)$ and $\bar{V}\left(\mathcal{P}_{\mathcal{I}^{\prime}}(\mathbb{L})\right)$. Hence, $D_{\mathcal{I}, \mathcal{I}^{\prime}}:=\alpha \circ \pi$ will be nonincreasing (specifically, either degree 0 or -2$)$ in the $(k=f)$-grading.

In the third case, $\operatorname{deg}_{f}\left(K^{\prime}\right)=0$, while $\operatorname{deg}_{f}\left(K_{i}\right)=\operatorname{deg}_{f}\left(K_{j}\right)=-2$. Since $\mathcal{P}_{\mathcal{I}^{\prime}}(\mathbb{L})$ has two fewer non-trivial components than $\mathcal{P}_{\mathcal{I}}(\mathbb{L})$, after applying the $f$-grading shift to $\bar{V}\left(\mathcal{P}_{\mathcal{I}}(\mathbb{L})\right)$ (resp., $\left.\bar{V}\left(\mathcal{P}_{\mathcal{I}^{\prime}}(\mathbb{L})\right)\right)$ to obtain $V\left(\mathcal{P}_{\mathcal{I}}(\mathbb{L})\right)$ (resp., $V\left(\mathcal{P}_{\mathcal{I}^{\prime}}(\mathbb{L})\right)$ ) as above, we similarly conclude that $D_{\mathcal{I}, \mathcal{I}^{\prime}}$ is non-increasing in the $(k=f)$-grading.

The case where $\mathcal{I}^{\prime}$ is obtained from $\mathcal{I}$ by a split saddle cobordism is completely analogous. 
Definition 2.22 Let $G C V(\mathcal{P}(\mathbb{L}))$ denote the associated graded complex of the $k$-filtered complex $C V(\mathcal{P}(\mathbb{L}))$. I.e.,

$$
G C V(\mathcal{P}(\mathbb{L})) \cong(C V(\mathcal{P}(\mathbb{L})), G D)
$$

where $G D=\sum_{\mathcal{I}, \mathcal{I}^{\prime}} G D_{\mathcal{I}, \mathcal{I}^{\prime}}$, with the sum taken over all pairs $\mathcal{I}, \mathcal{I}^{\prime} \in\{0,1\}^{\ell}$ where $\mathcal{I}^{\prime}$ is an immediate successor of $\mathcal{I}$ and

$$
G D_{\mathcal{I}, \mathcal{I}^{\prime}}: V\left(\mathcal{P}_{\mathcal{I}}(\mathbb{L})\right) \rightarrow V\left(\mathcal{P}_{\mathcal{I}^{\prime}}(\mathbb{L})\right)
$$

is given by $G \mathcal{V}_{m}$ (resp., $G \mathcal{V}_{\Delta}$ ) when $\mathcal{P}_{\mathcal{I}^{\prime}}(\mathbb{L})$ is obtained from $\mathcal{P}_{\mathcal{I}}(\mathbb{L})$ by a merge (resp., split) saddle cobordism. Let $V(\mathbb{L})$ denote the homology of $G C V(\mathcal{P}(\mathbb{L}))$.

Remark 2.23 Our chain complex $G C V(\mathcal{P}(\mathbb{L}))$ is isomorphic to the triply-graded chain complex $C^{* ; * * *}(\mathcal{P}(\overline{\mathbb{L}}))$ that Roberts associates to $\mathbb{L} \subset A \times I$ in [28]. Roberts' complex, in turn, is essentially equivalent to the Asaeda-Przytycki-Sikora chain complex for $\mathcal{P}(\mathbb{L})$, $\mathbb{L} \subset A \times I$, defined in [2], except that the Asaeda-Przytycki-Sikora construction requires $\mathbb{L}$ to be framed (but not oriented), whereas Roberts' construction requires $\mathbb{L}$ to be oriented (but not framed). Note that Asaeda-Przytycki-Sikora's $s$-grading appears (without loss of information) as Roberts' $f$-grading and that the relative $i$-and $j$-grading on $G C V(\mathcal{P}(\mathbb{L}))$, as well as the absolute $k$-grading, do not depend on the choice of an orientation for $\mathbb{L}$. Finally, we remark that the Khovanov-type homology of $\mathbb{L}, K h^{*}(\mathbb{L})$, referenced in the introduction and in the statements of Theorems 2.1 and 3.1, is $V(\overline{\mathbb{L}})$. See Remark 2.20.

\subsection{Sutured Floer Functor}

Given a link $\mathbb{L} \subset A \times I$, we alternatively obtain a filtered chain complex using sutured Floer homology (see [8]), a theory developed by Juhász which assigns Floer homology groups to balanced sutured manifolds. Recall ([8, Defn. 2.2]) that a sutured manifold $(Y, \Gamma)$ is said to be balanced if $\chi\left(R_{+}\right)=\chi\left(R_{-}\right)$, and the maps $\pi_{0}(\Gamma) \rightarrow \pi_{0}(\partial Y)$ and $\pi_{0}(\partial Y) \rightarrow \pi_{0}(Y)$ are surjective. Given a balanced sutured manifold $(Y, \Gamma)$-for example the branched doublecover, $\Sigma(A \times I, \mathbb{L})$, where $\mathbb{L} \subset A \times I$ is a link as above-one obtains its sutured Floer homology, $\operatorname{SFH}(Y, \Gamma)$, by the following procedure:

(1) Construct a balanced, sutured Heegaard diagram ([8, Defn. 2.7, 2.8]), ( $\Sigma, \boldsymbol{\alpha}, \boldsymbol{\beta})$, for $(Y, \Gamma)$, where $\Sigma$ is a compact, oriented surface with non-empty boundary and no closed components, and $\boldsymbol{\alpha}=\left\{\alpha_{1}, \ldots, \alpha_{d}\right\}, \boldsymbol{\beta}=\left\{\beta_{1}, \ldots, \beta_{d}\right\}$ are two sets of pairwise disjoint simple closed curves in $\operatorname{Int}(\Sigma)$ satisfying the condition that the set $\boldsymbol{\alpha}$ (resp., $\boldsymbol{\beta}$ ) is linearly independent in $H_{1}(\Sigma ; \mathbb{Z})$. Recall that one obtains a unique balanced, sutured manifold from a balanced, sutured Heegaard diagram by attaching 
3-dimensional 2-handles to $\Sigma \times I$ along the curves $\alpha_{i} \times\{0\}$ and $\beta_{j} \times\{1\}$ for $i, j \in\{1, \ldots d\} . \Gamma$ is $\partial \Sigma \times I$, and $s(\Gamma)=\partial \Sigma \times\left\{\frac{1}{2}\right\}$.

(2) The data of a balanced Heegaard diagram

$$
\left(\Sigma, \boldsymbol{\alpha}=\left\{\alpha_{1}, \ldots, \alpha_{d}\right\}, \boldsymbol{\beta}=\left\{\beta_{1}, \ldots, \beta_{d}\right\}\right)
$$

and a generic (family of) complex structures on $\Sigma$, is used to construct a Floer chain complex using the half-dimensional tori $\mathbb{T}_{\alpha}=\alpha_{1} \times \ldots \times \alpha_{d}$ and $\mathbb{T}_{\beta}=\beta_{1} \times \ldots \times \beta_{d}$ in $\operatorname{Sym}^{d}(\Sigma)$. Specifically, one obtains a chain complex with:

(a) Generators: $\left\{\mathbf{x} \in \mathbb{T}_{\alpha} \cap \mathbb{T}_{\beta}\right\}$,

(b) Differentials:

$$
\partial(\mathbf{x})=\sum_{\mathbf{y} \in \mathbb{T}_{\alpha} \cap \mathbb{T}_{\beta}} \sum_{\left\{\phi \in \pi_{2}(\mathbf{x}, \mathbf{y}) \mid \mu(\phi)=1\right\}} \widehat{\mathcal{M}}(\phi) \cdot \mathbf{y} .
$$

As usual, $\pi_{2}(\mathbf{x}, \mathbf{y})$ denotes the homotopy classes of disks (disjoint from $\partial \Sigma$ ) connecting $\mathbf{x}$ to $\mathbf{y}, \mu(\phi)$ denotes the Maslov index of $\phi \in \pi_{2}(\mathbf{x}, \mathbf{y})$, and $\widehat{\mathcal{M}}(\phi)$ denotes the moduli space of holomorphic representatives of $\phi$, modulo the standard $\mathbb{R}$ action.

(3) Denote by $\operatorname{CFH}(Y, \Gamma)$ the chain complex constructed in (2), and by $\operatorname{SFH}(Y, \Gamma)$ its homology.

In preparation for describing a sutured Floer-type "functor" analogous to the Khovanovtype "functor" defined in Section 2.2, we now establish, in Proposition 2.24, a relationship between the sutured Floer homology of $\Sigma(A \times I, \mathbb{L})$ and the knot Floer homology of a certain link in $\Sigma\left(S^{3}, \mathbb{L}\right)$. We shall see that $\operatorname{SFH}(\Sigma(A \times I, \mathbb{L}))$ can be identified as the homology of the associated graded complex of a natural filtered complex for this link in $\Sigma(A \times I, \mathbb{L})$. Proposition 2.24 provides a sutured-Floer theoretic interpretation of the Floer homology invariants studied in [28].

In what follows:

- $A \times I=\{(r, \theta, z) \mid r \in[1,2], \theta \in[0,2 \pi), z \in[0,1]\} \subset \mathbb{R}^{3} \cup \infty=S^{3}$ denotes the standard imbedding of $A \times I$ into $S^{3}$, with $\mathbb{L} \subset A \times I$ a fixed (isotopy class of) link,

- $B=\{(r, \theta, z) \mid r=0\} \cup \infty \subset S^{3}$ denotes a standardly-imbedded unknot,

- $\pi: \Sigma\left(S^{3}, \mathbb{L}\right) \rightarrow S^{3}$ denotes the branched-covering projection,

- $\widetilde{B}:=\pi^{-1}(B)$ denotes the preimage of $B$ in $\Sigma\left(S^{3}, \mathbb{L}\right)$,

- $D_{A} \times I:=\left\{(r, \theta, z) \in \mathbb{R}^{3} \mid r \in[0,2], z \in[0,1]\right\}$ denotes the product sutured manifold obtained by "capping off" one of the sutures of $A \times I$ as described in [6, Defn. 2.8, Prop. 6.2], 
- $i: A \times I \longrightarrow D_{A} \times I$ denotes the inclusion map,

- $\widehat{\mathbb{L}}:=i(\mathbb{L}) \subset D_{A} \times I$ denotes the image of $\mathbb{L}$ under the inclusion,

- $p(\mathbb{L}):=(l k(\mathbb{L}, B) \bmod 2)$ denotes the "mod 2 linking number" of $\mathbb{L}$ and $B$, which we will sometimes call the parity of $\mathbb{L}$,

- $\Theta$ denotes the bigraded $\mathbb{Z}_{2}$ vector space formally generated by $\theta^{+}, \theta^{-}$:

$$
\Theta:=\operatorname{Span}_{\mathbb{Z}_{2}}\left(\theta^{+}, \theta^{-}\right)
$$

with bigrading given by:

$$
\begin{aligned}
& \operatorname{deg}\left(\theta^{+}\right)=(0,0), \\
& \operatorname{deg}\left(\theta^{-}\right)=(-1,-1), \text { and }
\end{aligned}
$$

- $\widehat{\Theta}$ denotes the singly-graded $\mathbb{Z}_{2}$ vector space obtained by forgetting the first of the two gradings on $\Theta$.

We will also periodically use the following abbreviated notation:

$$
\begin{aligned}
Y_{\mathbb{L}} & :=\Sigma(A \times I, \mathbb{L}), \text { and } \\
Y_{\widehat{\mathbb{L}}} & :=\Sigma\left(D_{A} \times I, \widehat{\mathbb{L}}\right) .
\end{aligned}
$$

Proposition 2.24 $C F H\left(\Sigma\left(D_{A} \times I, \widehat{\mathbb{L}}\right)\right)$ can be given the structure of a filtered chain complex whose associated graded complex has homology

$$
\operatorname{SFH}(\Sigma(A \times I, \mathbb{L})) \cong \begin{cases}\widehat{H F K}\left(\Sigma\left(S^{3}, \mathbb{L}\right), \widetilde{B}\right) \otimes \Theta & \text { if } p(\mathbb{L})=1, \\ \widehat{H F K}\left(\Sigma\left(S^{3}, \mathbb{L}\right), \widetilde{B}\right) & \text { if } p(\mathbb{L})=0\end{cases}
$$

Moreover, the spectral sequence associated to this filtered complex converges to

$$
\operatorname{SFH}\left(\Sigma\left(D_{A} \times I, \widehat{\mathbb{L}}\right)\right) \cong \begin{cases}\widehat{H F}\left(\Sigma\left(S^{3}, \mathbb{L}\right)\right) \otimes \widehat{\Theta} & \text { if } p(\mathbb{L})=1, \text { and } \\ \widehat{H F}\left(\Sigma\left(S^{3}, \mathbb{L}\right)\right)\left\{\frac{1}{2}\right\} \otimes \widehat{\Theta} & \text { if } p(\mathbb{L})=0 .\end{cases}
$$

Remark 2.25 The bigrading referenced in the above proposition is the (A, M) bigrading associated to the null-homologous link $\widetilde{B} \subset \Sigma\left(S^{3}, \mathbb{L}\right)$, where $\mathbf{A}$ is the filtration (Alexander) grading on knot Floer homology defined in [17], [27] and elaborated in [21], [13] (see also [15], [3]), and $\mathbf{M}$ is the homological (Maslov) grading on Heegaard Floer homology defined in [24].

Note that an absolute $(\mathbf{A} \in \mathbb{Q}$ )-grading is only well-defined once a (homology class of Seifert surface has been chosen for $\widetilde{B}$, and an absolute $(\mathbf{M} \in \mathbb{Q}$ )-grading is only welldefined in torsion $\operatorname{Spin}^{c}$ structures of $\Sigma\left(S^{3}, \mathbb{L}\right)$. (The proof of) Proposition 2.24 asserts 
the existence of a canonical identification between filtered chain complexes, preserving relative bigradings. ${ }^{3}$ Therefore, $\operatorname{SFH}(\Sigma(A \times I, \mathbb{L}))$ inherits an $(\mathbf{A}, \mathbf{M})$-bigrading (resp., $\operatorname{SFH}\left(\Sigma\left(D_{A} \times I, \widehat{\mathbb{L}}\right)\right)$ inherits an $\mathbf{M}$-grading $)$, subject to these parameters.

Proof of Proposition 2.24 Note that $\Sigma(A \times I, \mathbb{L})$ is the sutured manifold $(Y, \Gamma)$ where

$$
Y=\Sigma\left(S^{3}, \mathbb{L}\right)-N(\widetilde{B}) \text { and } s(\Gamma)=\left\{\mu_{1}, \mu_{1}^{\prime}, \mu_{2}, \mu_{2}^{\prime}\right\},
$$

where the $\mu_{i}$ represent meridians of $\widetilde{B}$ and the $\mu_{i}^{\prime}$ represent oppositely-oriented meridians of $\widetilde{B}$. To see this, observe that $A \times I$ is the standard sutured knot complement of $B \subset S^{3}$, hence its preimage under $\pi$ is the complement of $\widetilde{B} \subset \Sigma\left(S^{3}, \mathbb{L}\right)$. It follows that each meridional (resp., oppositely-oriented meridional) suture lifts to two meridional (resp., oppositely-oriented meridional) sutures in $\Sigma(A \times I, \mathbb{L})$.

Furthermore:

- when $p(\mathbb{L})=0, \widetilde{B}=\widetilde{B}_{1} \amalg \widetilde{B}_{2}$ is a 2-component link. $(Y, \Gamma)$ is then the standard sutured link complement (see [8, Example 2.4]), with a pair, $\left(\mu_{i}, \mu_{i}^{\prime}\right)$, of sutures on each boundary component, $-\partial\left(N\left(\widetilde{B}_{i}\right)\right)$, and

- when $p(\mathbb{L})=1, \widetilde{B}$ is a 1 -component link. Hence, $(Y, \Gamma)$ is a sutured link complement with both pairs, $\left(\mu_{i}, \mu_{i}^{\prime}\right)$, of sutures on the single component of $\partial Y=-\partial(N(\widetilde{B}))$. Note that this is a non-standard sutured link complement, since there is an extra pair of sutures on the single component of $-\partial(N(\widetilde{B}))$.

Similarly, we see that $\Sigma\left(D_{A} \times I, \widehat{\mathbb{L}}\right)$ is obtained from $\Sigma\left(S^{3}, \mathbb{L}\right)$ by deleting two $B^{3}$ 's in $N(\widetilde{B})$. Put differently, one obtains $\Sigma\left(D_{A} \times I, \widehat{\mathbb{L}}\right)$ from $\Sigma(A \times I, \mathbb{L})=\Sigma\left(S^{3}, \mathbb{L}\right)-N(\widetilde{B})$ by filling in two $B^{3}$ 's in $N(\widetilde{B})$.

Now, suppose that $(\Sigma, \boldsymbol{\alpha}, \boldsymbol{\beta})$ is a sutured Heegaard diagram for $\Sigma(A \times I, \mathbb{L})$. Then the topological observations above imply that one obtains a 4-pointed Heegaard diagram

$$
\left(\Sigma^{\prime}, \boldsymbol{\alpha}^{\prime}, \boldsymbol{\beta}^{\prime}, \mathbb{O}=\left(O_{1}, O_{2}\right), \mathbb{X}=\left(X_{1}, X_{2}\right)\right)
$$

for $\Sigma\left(S^{3}, \mathbb{L}\right)$ compatible with the link $\widetilde{B}$ (in the sense of [17], [21], [13]), by taking

$$
\Sigma^{\prime}:=\Sigma \cup_{i=1,2} D_{O_{i}} \cup_{i=1,2} D_{X_{i}},
$$

where $D_{X_{i}}$ (resp., $D_{O_{i}}$ ) are disks containing a distinguished basepoint $X_{i}$ (resp., $O_{i}$ ) filling in the boundary components of $\Sigma$ corresponding to the meridians (resp., oppositely-oriented meridians) of $\widetilde{B}$,

\footnotetext{
${ }^{3}$ With respect to a fixed Seifert surface, relative $\mathbf{A}$-gradings lie in $\mathbb{Z}$, while in a fixed $\operatorname{Spin}^{c}$ structure, relative $\mathbf{M}$-gradings lie in some quotient of $\mathbb{Z}$. See [17, Sec. 2], [19, Sec.4].
} 
- $\boldsymbol{\alpha}^{\prime}$ (resp., $\boldsymbol{\beta}^{\prime}$ ), are the images of $\boldsymbol{\alpha}$ (resp., $\boldsymbol{\beta}$ ) under the inclusion map $\Sigma \rightarrow \Sigma^{\prime}$.

Similarly, by gluing in only the disks $D_{X_{1}}, D_{X_{2}}$ to $(\Sigma, \boldsymbol{\alpha}, \boldsymbol{\beta})$, one obtains a sutured Heegaard diagram, $(\widehat{\Sigma}, \widehat{\boldsymbol{\alpha}}, \widehat{\boldsymbol{\beta}})$, for $\Sigma\left(D_{A} \times I, \widehat{\mathbb{L}}\right)$.

Let $\mathcal{C}^{\prime}$ represent the Heegaard-Floer chain complex associated to $\left(\Sigma^{\prime}, \boldsymbol{\alpha}^{\prime}, \boldsymbol{\beta}^{\prime}, \mathbb{O}, \mathbb{X}\right)$ with

- Generators: $\left\{\mathbf{x} \in \mathbb{T}_{\alpha^{\prime}} \cap \mathbb{T}_{\beta^{\prime}}\right\}$

- Differential:

$$
\partial^{\prime}(\mathbf{x})=\sum_{\mathbf{y} \in \mathbb{T}_{\alpha^{\prime}} \cap \mathbb{T}_{\beta^{\prime}}} \sum_{\left\{\phi \in \pi_{2}(\mathbf{x}, \mathbf{y})\right.} \widehat{M}(\phi) \cdot \mathbf{y},
$$

where $n_{\mathbb{O}}(\phi):=\sum_{i=1}^{2} n_{O_{i}}(\phi)$. Then the sutured Floer chain complex associated to $(\widehat{\Sigma}, \widehat{\boldsymbol{\alpha}}, \widehat{\boldsymbol{\beta}})$ is canonically isomorphic to $\mathcal{C}^{\prime},{ }^{4}$ since the condition that $n_{\mathbb{O}}(\phi)=0$ for $\phi \subset \Sigma^{\prime}$ is equivalent to the condition that $\phi \subset \widehat{\Sigma}$ is never adjacent to $\partial \widehat{\Sigma}$. Hence,

$$
\operatorname{SFH}\left(\Sigma\left(D_{A} \times I, \mathbb{L}\right)\right) \cong \begin{cases}\widehat{H F}\left(\Sigma\left(S^{3}, \mathbb{L}\right)\right) \otimes \widehat{\Theta} & \text { if } p(\mathbb{L})=1, \text { and } \\ \widehat{H F}\left(\Sigma\left(S^{3}, \mathbb{L}\right)\right)\left\{\frac{1}{2}, \frac{1}{2}\right\} \otimes \widehat{\Theta} & \text { if } p(\mathbb{L})=0,\end{cases}
$$

as desired. The extra $\left\{\frac{1}{2}, \frac{1}{2}\right\}$ grading shift when $p(\mathbb{L})=0$ arises due to the convention that $\widehat{H F K}(Y, L)$, for $L$ an $\ell$-component link, is defined to be the knot Floer homology of the "knotification" of $L$ in $Y \#^{\ell-1}\left(S^{1} \times S^{2}\right)$ (see [17, Sec. 2.1]).

Furthermore, since intersection positivity guarantees that $n_{\mathbb{X}}(\phi) \geq 0$ for all holomorphic $\phi \in \pi_{2}(\mathbf{x}, \mathbf{y})$, we can endow $\mathcal{C}^{\prime}$ with the structure of a $\mathbb{Z}$-filtered complex by decomposing the differential as

$$
\partial^{\prime}=\sum_{i=0}^{\infty} \partial_{i}^{\prime}
$$

where

$$
\partial_{i}^{\prime}(\mathbf{x}):=\sum_{\mathbf{y} \in \mathbb{T}_{\alpha^{\prime}} \cap \mathbb{T}_{\beta^{\prime}}} \sum_{\left\{\phi \in \pi_{2}(\mathbf{x}, \mathbf{y}) \mid \mu(\phi)=1, n_{\mathbb{O}}(\phi)=0, n_{\mathbb{X}}(\phi)=i\right\}} \widehat{M}(\phi) \cdot \mathbf{y} .
$$

The associated graded complex, $\left(\mathcal{C}^{\prime}, \partial_{0}^{\prime}\right)$, is therefore chain isomorphic to the sutured Floer complex obtained from $(\Sigma, \boldsymbol{\alpha}, \boldsymbol{\beta})$, since counting $\phi \subset \Sigma^{\prime}$ with $n_{\mathbb{X}}(\phi)=n_{\mathbb{O}}(\phi)=0$ is equivalent to counting $\phi \subset \Sigma$ disjoint from $\partial \Sigma$. Hence, $\operatorname{SFH}(\Sigma(A \times I, \mathbb{L}))$ is the homology of the associated graded complex of the filtered complex $C F H\left(\Sigma\left(D_{A} \times I, \widehat{\mathbb{L}}\right)\right)$.

\footnotetext{
${ }^{4}$ after fixing a generic choice of almost complex structure on $\widehat{\Sigma}$, which extends uniquely to a generic choice of almost complex structure on $\Sigma^{\prime}$
} 
This immediately implies (see [8, Examples 2.3, 2.4], [8, Prop. 9.2], and [21, Thm. 1.1], combined with [14, Lemma 2.12]):

$$
\operatorname{SFH}(\Sigma(A \times I, \mathbb{L})) \cong \begin{cases}\widehat{H F K}\left(\Sigma\left(S^{3}, \mathbb{L}\right), \widetilde{B}\right) \otimes \Theta & \text { if } p=1, \\ \left.\widehat{H F K}\left(\Sigma\left(S^{3}, \mathbb{L}\right)\right), \widetilde{B}\right) & \text { if } p=0\end{cases}
$$

as desired.

We are now ready to define a sutured Floer-type "functor".

In what follows, let $\left(\mathbb{L}=\mathbb{L}_{\text {triv }} \amalg \mathbb{L}_{\text {non }}\right) \subset A \times I$ be a resolved link with

$$
\begin{aligned}
& \mathbb{L}_{\text {triv }}=K_{1} \amalg \ldots \amalg K_{t}, \text { and } \\
& \mathbb{L}_{\text {non }}=K_{t+1} \amalg \ldots \amalg K_{t+n},
\end{aligned}
$$

and $\widehat{\mathbb{L}}=i(\mathbb{L}) \subset\left(D_{A} \times I\right)$ its image under the inclusion $i: A \times I \rightarrow D_{A} \times I$ as before.

Definition 2.26 Let $Z_{H}(\mathbb{L})$ denote the $\mathbb{Z}_{2}$-vector space formally generated by $\left[K_{1}\right], \ldots,\left[K_{t+n}\right]$ :

$$
Z_{H}(\mathbb{L}):=\operatorname{Span}_{\mathbb{Z}_{2}}\left(\left[K_{1}\right], \ldots,\left[K_{t+n}\right]\right),
$$

endowed with the bigrading:

$$
\operatorname{deg}\left(\left[K_{i}\right]\right):= \begin{cases}(0,-1) & \text { for } 1 \leq i \leq t, \text { and } \\ (-1,-1) & \text { else. }\end{cases}
$$

The space $Z_{H}(\mathbb{L})$ decomposes as $Z_{H}\left(\mathbb{L}_{\text {triv }}\right) \oplus Z_{H}\left(\mathbb{L}_{\text {non }}\right)$, where

$$
\begin{aligned}
Z_{H}\left(\mathbb{L}_{\text {triv }}\right) & :=\operatorname{Span}_{\mathbb{Z}_{2}}\left(\left[K_{1}\right], \ldots,\left[K_{t}\right]\right), \\
Z_{H}\left(\mathbb{L}_{\text {non }}\right) & :=\operatorname{Span}_{\mathbb{Z}_{2}}\left(\left[K_{t+1}\right], \ldots,\left[K_{t+n}\right]\right) .
\end{aligned}
$$

Definition 2.27 Let $\bar{V}_{H}(\mathbb{L})$ denote the exterior algebra

$$
\bar{V}_{H}(\mathbb{L}):=\Lambda^{*} Z_{H}(\mathbb{L}),
$$

with its bigrading induced from $Z_{H}$ :

$$
\operatorname{deg}\left(\left[K_{i_{1}}\right] \wedge \ldots \wedge\left[K_{i_{k}}\right]\right):=\operatorname{deg}\left(\left[K_{i_{1}}\right]\right)+\ldots+\operatorname{deg}\left(\left[K_{i_{k}}\right]\right),
$$

as in Definition 2.14, and let $V_{H}(\mathbb{L})$ denote the following normalization of $\bar{V}_{H}(\mathbb{L})$ :

$$
V_{H}(\mathbb{L}):=\bar{V}_{H}(\mathbb{L})\left\{\frac{n-p(\mathbb{L})}{2}, \frac{t+n-p(\mathbb{L})}{2}\right\} .
$$


Recall that $p(\mathbb{L}):=l k(\mathbb{L}, B) \bmod 2$. In particular, when $\mathbb{L}$ is a resolved link,

$$
p(\mathbb{L})=n \bmod 2 .
$$

Proposition 2.28 Let $\mathbb{L} \subset A \times I$ be a resolved link, as above. Then

$$
\operatorname{SFH}\left(Y_{\mathbb{L}}\right) \cong V_{H}(\mathbb{L})
$$

as an $(\mathbf{A}, \mathbf{M})$-bigraded $\mathbb{Z}_{2}$ vector space.

Proof Recall (see [19, Sec. 4.2.5], [6, Sec. 3.4]) that if $(Y, \Gamma)$ is a balanced sutured manifold, then $\operatorname{SFH}(Y, \Gamma)$ admits an action of $\Lambda^{*}\left(H_{1}^{\circ}(Y, \partial Y)\right)$, where here (and in what follows), we use $H_{*}^{\circ}(-)$ to denote $H_{*}(-; \mathbb{Z}) /$ Tors.

Since Proposition 2.24 tells us that $\operatorname{SFH}\left(Y_{\mathbb{L}}\right)$ is the homology of the associated graded complex of $C F H\left(Y_{\widehat{\mathbb{L}}}\right)$, it will be convenient for us to begin by examining $\operatorname{SFH}\left(Y_{\widehat{\mathbb{L}}}\right)$.

In particular, [6, Lem. 5.16] tells us that $\operatorname{SFH}\left(Y_{\widehat{\mathbb{L}}}\right)$ is a free, rank 1 module over

$$
\Lambda^{*}\left(H_{1}^{\circ}\left(Y_{\widehat{\mathbb{L}}}, \partial Y_{\widehat{\mathbb{L}}}\right) \otimes_{\mathbb{Z}} \mathbb{Z}_{2}\right)
$$

Furthermore, in the proof of $[6$, Lem. 5.16] we see that

$$
\begin{aligned}
H_{1}^{\circ}\left(Y_{\widehat{\mathbb{L}}}, \partial Y_{\widehat{\mathbb{L}}}\right) \otimes_{\mathbb{Z}} \mathbb{Z}_{2} & \cong H^{2}\left(\#^{t+n} S^{1} \times S^{2} ; \mathbb{Z}_{2}\right) \\
& \cong Z_{H}(\mathbb{L}),
\end{aligned}
$$

as ungraded vector spaces and hence

$$
\Lambda^{*}\left(H_{1}^{\circ}\left(Y_{\widehat{\mathbb{L}}}, \partial Y_{\widehat{\mathbb{L}}}\right) \otimes_{\mathbb{Z}} \mathbb{Z}_{2}\right) \cong \bar{V}_{H}(\mathbb{L})
$$

as ungraded algebras. To see that they agree as bigraded algebras-and, hence, that $\operatorname{SFH}\left(Y_{\mathbb{L}}\right) \cong V_{H}(\mathbb{L})$ up to an overall bigrading shift-we need only show that under the identification:

$$
\phi: Z_{H}(\mathbb{L}) \rightarrow H_{1}^{\circ}\left(Y_{\widehat{\mathbb{L}}}, \partial Y_{\widehat{\mathbb{L}}}\right) \otimes_{\mathbb{Z}} \mathbb{Z}_{2}
$$

elements of

$$
\left.Z_{H}\left(\mathbb{L}_{\text {triv }}\right) \leq Z_{H}(\mathbb{L}) \text { (resp., of } Z_{H}\left(\mathbb{L}_{\text {non }}\right) \leq Z_{H}(\mathbb{L})\right)
$$

are sent to algebra generators of $(\mathbf{A}, \mathbf{M})$-degree $(0,-1)$, (resp., $(-1,-1))$.

Since it is immediate, by definition of the $H_{1}^{\circ}$ action (see [19, Sec. 4.2.5], [6, Sec. 3.4]), that the image of each element of $Z_{H}(\mathbb{L})$ has $\mathbf{M}$-degree -1 , as desired, we are left to verify that the $\mathbf{A}$-degrees of elements of $Z_{H}(\mathbb{L})$ are as stated.

To see this, we will study the $H_{1}^{\circ}$ action on a chain complex, $C F H\left(Y_{\widehat{\mathbb{L}}}\right)$, associated to a particularly nice sutured Heegaard diagram for $Y_{\widehat{\mathbb{L}}}$. The generators of $C F H\left(Y_{\widehat{\mathbb{L}}}\right)$ will have 

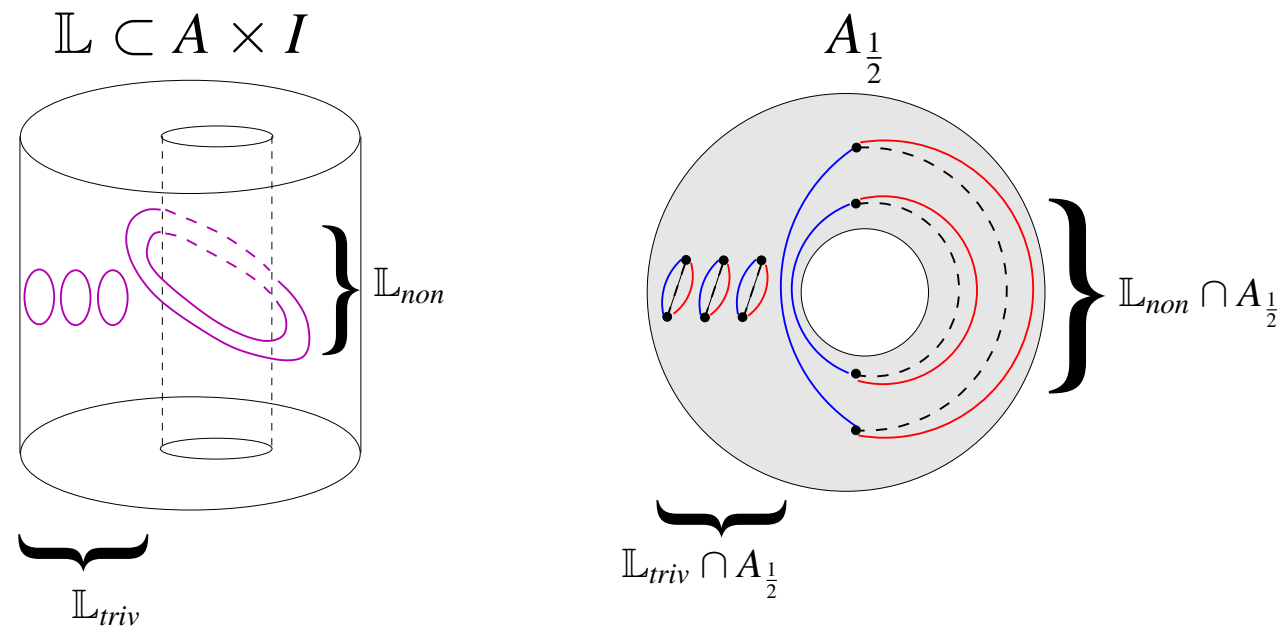

Figure 3: The lefthand figure shows $\left(\mathbb{L}=\mathbb{L}_{\text {triv }} \amalg \mathbb{L}_{\text {non }}\right)$ standardly imbedded in $A \times I$. The righthand figure shows the middle level, $A_{\frac{1}{2}}$. The black dots represent the intersection points, $\mathbb{L} \cap A_{\frac{1}{2}}$, the dotted lines are the branch cuts, and the red (resp., blue) arcs are the projections of the cups (resp., caps).

well-defined absolute $\mathbf{A}$-gradings, and the homology of the associated graded complex will be $\operatorname{SFH}\left(Y_{\mathbb{L}}\right)$, as in Proposition 2.24.

Accordingly, we proceed as in [6, Sec. 5.3] by choosing an isotopy class representative of $\mathbb{L}$ satisfying $K_{i} \pitchfork A_{\frac{1}{2}} \neq \emptyset$ for all $i \in\{1, \ldots t+n\}$, where $A_{\frac{1}{2}}:=A \times\left\{\frac{1}{2}\right\}$.

Let $\pi: Y_{\mathbb{L}} \rightarrow A \times I$ be the double-branched covering projection, and $\pi_{\frac{1}{2}}: \pi^{-1}\left(A_{\frac{1}{2}}\right) \rightarrow A_{\frac{1}{2}}$ its restriction to the $\frac{1}{2}$ level. Then $(\Sigma, \boldsymbol{\alpha}, \boldsymbol{\beta})$ is a sutured Heegaard diagram for $Y_{\mathbb{L}}$, where

- $\Sigma=\pi_{\frac{1}{2}}^{-1}\left(A_{\frac{1}{2}}\right)$

- $\boldsymbol{\alpha}=\pi_{\frac{1}{2}}^{-1}(\mathcal{P}(\mathrm{cups}))$

- $\boldsymbol{\beta}=\pi_{\frac{1}{2}}^{-1}(\mathcal{P}$ (caps) $)$

See Figures 3 and 4 along with [6, Sec. 5.3] for more details.

Furthermore, as described in the proof of Proposition 2.24, we obtain a sutured Heegaard diagram, $(\widehat{\Sigma}, \widehat{\boldsymbol{\alpha}}, \widehat{\boldsymbol{\beta}})$, for $Y_{\widehat{\mathbb{L}}}=\Sigma\left(D_{A} \times I, \mathbb{L}\right)$ by gluing in disks $D_{X_{1}}, D_{X_{2}}$ to two of the boundary components. Let

$$
\begin{aligned}
\widehat{\boldsymbol{\alpha}} & =\left\{\widehat{\alpha}_{1}, \ldots, \widehat{\alpha}_{t}, \widehat{\alpha}_{t+1}, \ldots \widehat{\alpha}_{t+n}\right\}, \text { and } \\
\widehat{\boldsymbol{\beta}} & =\left\{\widehat{\beta}_{1}, \ldots, \widehat{\beta}_{t}, \widehat{\beta}_{t+1}, \ldots, \widehat{\beta}_{t+n}\right\}
\end{aligned}
$$



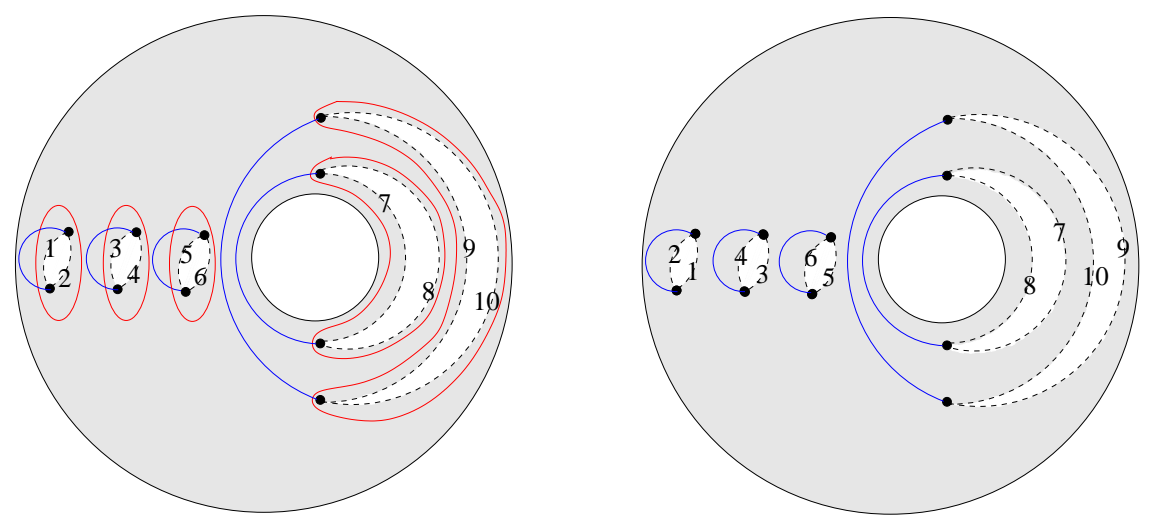

Figure 4: A sutured Heegaard diagram, $(\Sigma, \boldsymbol{\alpha}, \boldsymbol{\beta})$, for $Y_{\mathbb{L}}$, where $\mathbb{L}$ is as in Figure 3 . The dotted lines are identified as labeled, and the colored circles are isotopic to the preimages of the colored arcs in Figure 3.

be labeled as in Figure 5.

Since

$$
\alpha_{i} \cap \beta_{j}=\widehat{\alpha}_{i} \cap \widehat{\beta}_{j}= \begin{cases}\left\{\left(v_{+}\right)_{i},\left(v_{-}\right)_{i}\right\} & \text { if } i=j \\ \emptyset & \text { otherwise }\end{cases}
$$

we obtain the following set of $\mathbb{Z}_{2}$-module generators for both $C F H\left(Y_{\mathbb{L}}\right)$ and $C F H\left(Y_{\widehat{\mathbb{L}}}\right)$ :

$$
\left\{\left(v_{ \pm}\right)_{1} \otimes \cdots \otimes\left(v_{ \pm}\right)_{t+n}\right\} .
$$

Furthermore, as in the proof of [6, Lem. 5.18], $H_{1}^{\circ}\left(Y_{\widehat{\mathbb{L}}}, \partial Y_{\widehat{\mathbb{L}}}\right)$ has a basis whose elements, $\left\{\left[K_{i}\right]\right\}_{i=1}^{t+n}$, are in one-to-one correspondence with the components, $\left\{K_{i}\right\}_{i=1}^{t+n}$, of $\mathbb{L}$, and the $H_{1}^{\circ}$-action on the filtered complex, $C F H\left(Y_{\widehat{\mathbb{L}}}\right)$ is extended linearly from the following action on generators:

Let $x_{i} \in\left\{\left(v_{+}\right)_{i},\left(v_{-}\right)_{i}\right\}$ for all $i \in\{1, \ldots, t+n\}$, then

$$
\left[K_{j}\right] \cdot\left(x_{1} \otimes \ldots \otimes x_{t+n}\right):= \begin{cases}x_{1} \otimes \ldots \otimes\left(v_{-}\right)_{j} \otimes \ldots \otimes x_{t+n} & \text { if } x_{j}=\left(v_{+}\right)_{j}, \text { and } \\ 0 & \text { if } x_{j}=\left(v_{-}\right)_{j} .\end{cases}
$$

Recalling that if $\mathbf{x}, \mathbf{y} \in \mathbb{T}_{\widehat{\alpha}} \cap \mathbb{T}_{\widehat{\beta}}$ and $\phi \in \pi_{2}(\mathbf{x}, \mathbf{y})$, the relative $\mathbf{A}$ grading is given by:

$$
\mathbf{A}(\mathbf{x})-\mathbf{A}(\mathbf{y})=n_{\mathbb{X}}(\phi)
$$

where

$$
n_{\mathbb{X}}(\phi):=\sum_{i=1}^{2} n_{X_{i}}(\phi),
$$

there are two cases to consider. 

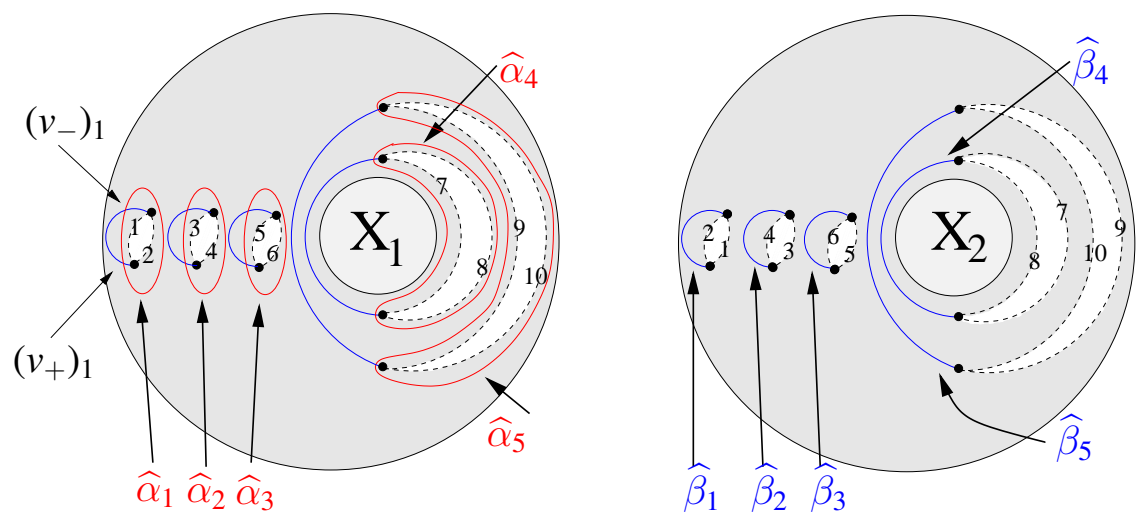

Figure 5: A sutured Heegaard diagram for $Y_{\widehat{\mathbb{L}}}$. The $\widehat{\alpha}, \widehat{\beta}$ curves are numbered in increasing order from left to right (resp., from inside to outside) in the $\mathbb{L}_{\text {triv }}$ (resp., the $\mathbb{L}_{\text {non }}$ ) section of $\widehat{\Sigma}$.

Case 1: $K_{j} \in \mathbb{L}_{\text {triv }}$

Let $x_{i} \in\left\{\left(v_{+}\right)_{i},\left(v_{-}\right)_{i}\right\}$ for all $i=1, \ldots, t+n .\left[K_{j}\right]$ acts non-trivially on a generator, $\mathbf{x}$, iff it is of the form:

$$
\mathbf{x}=x_{1} \otimes \ldots \otimes\left(v_{+}\right)_{j} \otimes \ldots \otimes x_{t+n},
$$

in which case:

$$
\left[K_{j}\right] \cdot(\mathbf{x})=x_{1} \otimes \ldots \otimes\left(v_{-}\right)_{j} \otimes \ldots \otimes x_{t+n} .
$$

Since $\mathbf{x}$ and $\left[K_{j}\right] \cdot(\mathbf{x})$ are connected by a domain, $\phi$, as in Figure 6 , and $\phi$ satisfies $n_{\mathbb{X}}(\phi)=0$, we conclude that

$$
\mathbf{A}(\mathbf{x})-\mathbf{A}\left(\left[K_{j}\right] \cdot \mathbf{x}\right)=0
$$

as desired.

Case 2: $K_{j} \in \mathbb{L}_{\text {non }}$

As in Case 1, above, $\left[K_{j}\right]$ acts non-trivially on a generator, $\mathbf{x}$, iff it is of the form:

$$
\mathbf{x}=x_{1} \otimes \ldots \otimes\left(v_{+}\right)_{j} \otimes \ldots \otimes x_{t+n}
$$

in which case:

$$
\left[K_{j}\right] \cdot(\mathbf{x})=x_{1} \otimes \ldots \otimes\left(v_{-}\right)_{j} \otimes \ldots \otimes x_{t+n} .
$$

When $K_{j} \in \mathbb{L}_{n o n}$, then $\mathbf{x}$ and $\left[K_{j}\right] \cdot(\mathbf{x})$ are connected by a domain, $\phi$, as in Figure 7. Since $\phi$ satisfies $n_{\mathbb{X}}(\phi)=1$,

$$
\mathbf{A}(\mathbf{x})-\mathbf{A}\left(\left[K_{j}\right] \cdot \mathbf{x}\right)=1,
$$




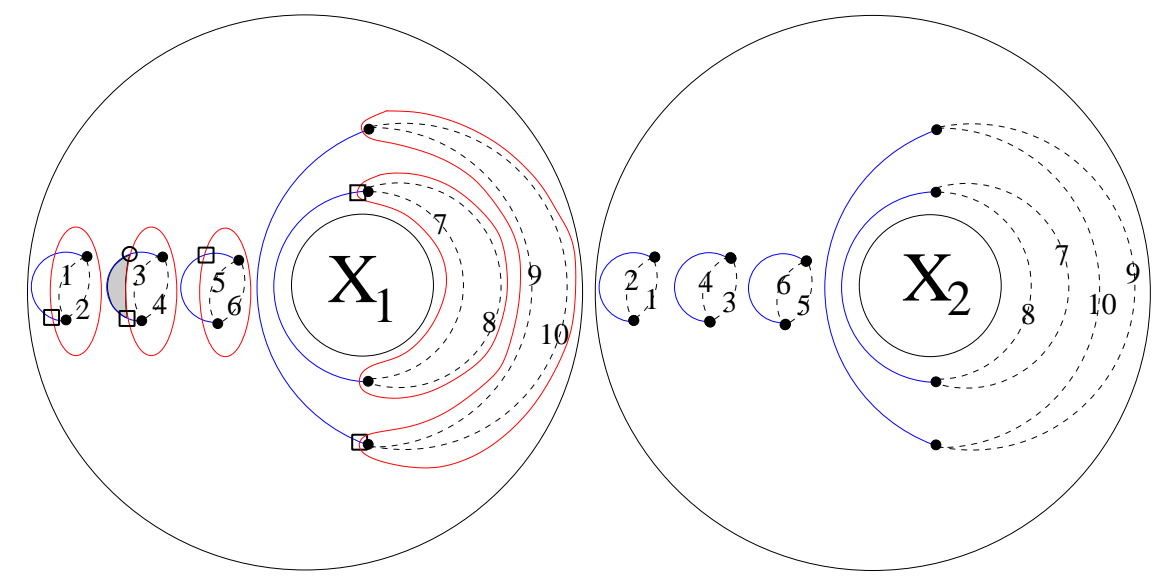

Figure 6: The shaded region represents a domain, $\phi$, connecting two generators, $\mathbf{x}$ and $\mathbf{y}$, in $(\widehat{\Sigma}, \widehat{\boldsymbol{\alpha}}, \widehat{\boldsymbol{\beta}})$. Here $\mathbf{x}$ is represented by the square intersection point(s), while $\mathbf{y}$ agrees with $\mathbf{x}$ everywhere except at $\widehat{\alpha}_{2} \cap \widehat{\beta}_{2}$, where $\mathbf{y}$ is represented by the circular intersection point.

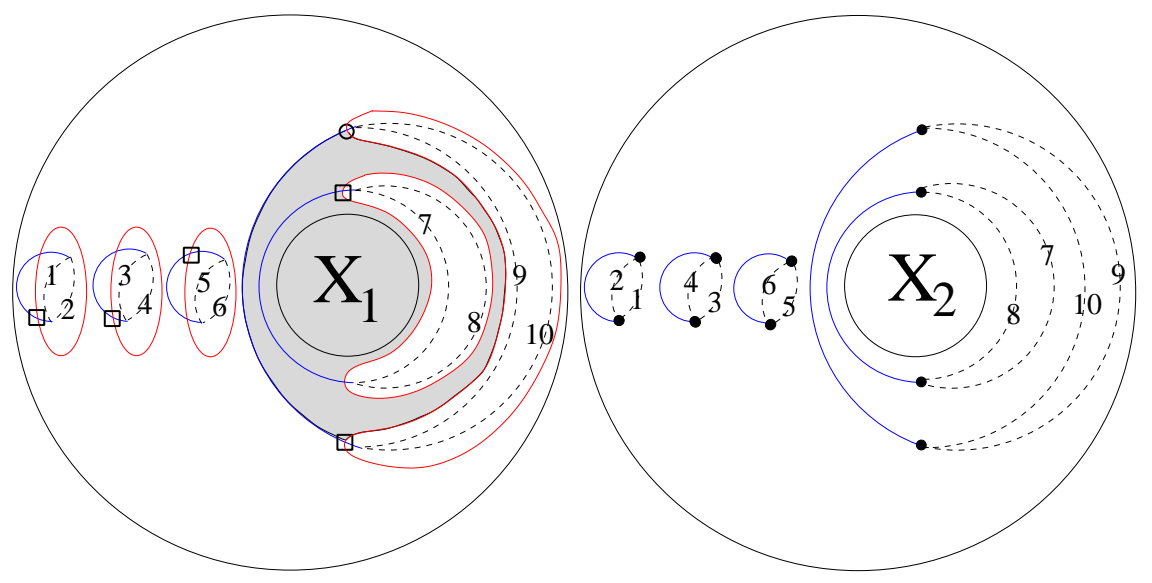

Figure 7: The shaded region represents a domain, $\phi$, connecting two generators, $\mathbf{x}$ and $\mathbf{y}$, in $(\widehat{\Sigma}, \widehat{\boldsymbol{\alpha}}, \widehat{\boldsymbol{\beta}})$. Here, $\mathbf{x}$ is represented by the square intersection point(s), while $\mathbf{y}$ agrees with $\mathbf{x}$ everywhere except at $\widehat{\alpha}_{5} \cap \widehat{\beta}_{5}$, where $\mathbf{y}$ is represented by the circular intersection point. 
as desired.

We conclude that, with respect to this choice of sutured Heegaard diagram,

$$
C F H\left(Y_{\mathbb{L}}\right) \cong V_{H}(\mathbb{L})
$$

as relatively $(\mathbf{A}, \mathbf{M})$ bigraded $\mathbb{Z}_{2}$ modules, and, hence, that $\operatorname{SFH}\left(Y_{\mathbb{L}}\right) \cong V_{H}(\mathbb{L})$ as relatively (A, M) bigraded modules, since:

$$
2^{t+n}=r k\left(C F H\left(Y_{\mathbb{L}}\right)\right) \geq r k\left(\operatorname{SFH}\left(Y_{\mathbb{L}}\right)\right) \geq r k\left(\operatorname{SFH}\left(Y_{\widehat{\mathbb{L}}}\right)\right)=2^{t+n} .
$$

Note that the second inequality above arises because $C F H\left(Y_{\mathbb{L}}\right)$ can be identified as the associated graded complex of the filtered complex $C F H\left(Y_{\widehat{\mathbb{L}}}\right)$, as in Proposition 2.24.

To see that the absolute $\mathbf{A}$ and $\mathbf{M}$ gradings on $\operatorname{SFH}\left(Y_{\mathbb{L}}\right)$ are as stated, it suffices to show that the extremal generator,

$$
\mathbf{v}_{+}:=\left(v_{+}\right)_{1} \otimes \ldots \otimes\left(v_{+}\right)_{t+n}
$$

has bigrading

$$
(\mathbf{A}, \mathbf{M})=\left(\frac{n-p(\mathbb{L})}{2}, \frac{t+n-p(\mathbb{L})}{2}\right)
$$

To see that $\mathbf{A}\left(\mathbf{v}_{+}\right)$is as claimed, use Proposition 2.24, combined with the symmetry properties of knot Floer homology with extra basepoint pairs [17, Sec. 3.5], [21, Sec. 8], [14, Lem. 2.12], which tell us that for a multiply-pointed Heegaard diagram associated to an $\ell$ component link $K=\amalg_{i=1}^{\ell} K_{i}$ for which there are $n_{i}(\mathbb{O}, \mathbb{X})$ basepoint pairs associated to the link component $K_{i}$, we have:

$$
\left(\widehat{H F K}(Y, K) \otimes \Theta^{\otimes s}\right)_{(a, m)} \cong\left(\widehat{H F K}(Y, K) \otimes \Theta^{\otimes s}\right)_{(-a-s, m-2 a-s)}
$$

where $s:=\sum_{i=1}^{\ell}\left(n_{i}-1\right), \Theta$ is the bigraded $\mathbb{Z}_{2}$ vector space generated by $\theta^{+}, \theta^{-}$satisfying

$$
\begin{aligned}
& \left(\mathbf{A}\left(\theta^{+}\right), \mathbf{M}\left(\theta^{+}\right)\right)=(0,0), \\
& \left(\mathbf{A}\left(\theta^{-}\right), \mathbf{M}\left(\theta^{-}\right)\right)=(-1,-1),
\end{aligned}
$$

and $\left(\widehat{H F K}(Y, K) \otimes \Theta^{\otimes s}\right)_{(a, m)}$ denotes the summand in $(\mathbf{A}, \mathbf{M})$ bigrading $(a, m)$.

Since the above symmetry in the case of interest $\left(Y=\Sigma\left(S^{3}, \mathbb{L}\right), K=\widetilde{B}\right)$ identifies $\mathbf{v}_{+}$with $\mathbf{v}_{-}=\left(v_{+}\right)_{1} \otimes \ldots\left(v_{+}\right)_{t} \otimes\left(v_{-}\right)_{t+1} \otimes \ldots \otimes\left(v_{-}\right)_{t+n}$, we obtain

$$
\begin{aligned}
\mathbf{A}\left(\mathbf{v}_{+}\right)-\mathbf{A}\left(\mathbf{v}_{-}\right) & =\mathbf{A}\left(\mathbf{v}_{+}\right)-\left(-\mathbf{A}\left(\mathbf{v}_{+}\right)-s\right) \\
n & =2 \mathbf{A}\left(\mathbf{v}_{+}\right)+s \\
\frac{n-s}{2} & =\mathbf{A}\left(\mathbf{v}_{+}\right) \\
\frac{n-p(\mathbb{L})}{2} & =\mathbf{A}\left(\mathbf{v}_{+}\right)
\end{aligned}
$$


as desired.

To see that

$$
\mathbf{M}\left(\mathbf{v}_{+}\right)=\frac{t+n-p(\mathbb{L})}{2}
$$

we use the fact that

$$
\Sigma\left(S^{3}, \mathbb{L}\right)=\#^{(t+n)-1}\left(S^{1} \times S^{2}\right),
$$

combined with the fact that, by convention, the knot Floer homology of a multi-component link in $Y$ comes from the "knotification" of the link [17, Sec. 2.1] in $Y \#(\ell-1) S^{1} \times S^{2}$. Using the Heegaard Floer Künneth principle ([18, Thm. 1.5]) and the fact (cf. [19, Subsec. 9.1]) that $\widehat{H F}\left(S^{1} \times S^{2}\right)$ is the free $\mathbb{Z}_{2}$-module on generators $\left\{\mathbf{w}_{+}, \mathbf{w}_{-}\right\}$with

$$
\mathbf{M}\left(\mathbf{w}_{ \pm}\right)= \pm \frac{1}{2}
$$

implies that

$$
\mathbf{M}\left(\mathbf{v}_{+}\right)=\frac{t+n-p(\mathbb{L})}{2}
$$

as desired.

The following proposition establishes the equivalence of the Khovanov and sutured Floer "functors" on resolved links, $\mathbb{L} \subset A \times I$. In what follows,

- $V(\mathbb{L})\left(\right.$ resp., $\left.V_{H}(\mathbb{L})\right)$ denotes the $(f, q)$-bigraded (resp., $(\mathbf{A}, \mathbf{M})$-bigraded) vector space of Definition 2.14 (resp., Definition 2.27).

- For resolved links $\mathbb{L}^{\prime}, \mathbb{L}^{\prime \prime} \subset A \times I$ connected by a saddle cobordism $S, G \mathcal{F}_{S}$ denotes the map

$$
\operatorname{SFH}\left(Y_{\mathbb{L}^{\prime}}\right) \rightarrow \operatorname{SFH}\left(Y_{\mathbb{L}^{\prime \prime}}\right)
$$

induced by performing surgery on the corresponding knot in $Y_{\mathbb{L}^{\prime}}$ as in [6, Sec. 4].

- $G \mathcal{V}_{S}$ denotes the $f$-grading-preserving part of the map induced by the saddlecobordism $S$, as in Definitions 2.15 and 2.16.

Proposition 2.29 For each resolved link

$$
\mathbb{L}=\left(\mathbb{L}_{\text {triv }} \amalg \mathbb{L}_{\text {non }}\right) \subset A \times I
$$

there is a canonical isomorphism

$$
\Psi_{\mathbb{L}}: V(\mathbb{L}) \rightarrow S F H\left(Y_{\mathbb{L}}\right)
$$


Furthermore, this isomorphism is natural in the sense that if $\mathbb{L}^{\prime}, \mathbb{L}^{\prime \prime} \subset A \times I$ are resolved links and $S: \mathbb{L}^{\prime} \rightarrow \mathbb{L}^{\prime \prime}$ is a saddle cobordism, the following diagram commutes:

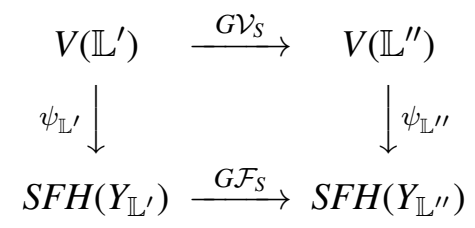

Proof Let $\mathbb{L} \subset A \times I$ be any resolved link. Proposition 2.28 tells us that $S F H\left(Y_{\mathbb{L}}\right) \cong V_{H}(\mathbb{L})$, so the canonical isomorphism, $\Psi_{\mathbb{L}}: V(\mathbb{L}) \rightarrow S F H\left(Y_{\mathbb{L}}\right)$ for any resolved $\mathbb{L} \subset A \times I$ is the one induced by the canonical isomorphism $Z(\mathbb{L}) \rightarrow Z_{H}(\mathbb{L})$.

Now denote by $\widehat{\mathbb{L}}$ the image of $\mathbb{L}$ under the inclusion $i: A \times I \longrightarrow D_{A} \times I$. Then $\widehat{\mathbb{L}}$ is an admissible, 0-balanced, resolved tangle in $D_{A} \times I$, in the sense of [6, Defn. 5.1-5.5], so [6, Prop. 5.17] gives us an isomorphism

$$
\psi_{\widehat{\mathbb{L}}}: V(\widehat{\mathbb{L}}) \longrightarrow \operatorname{SFH}\left(Y_{\widehat{\mathbb{L}}}\right)
$$

such that if $\mathbb{L}^{\prime}, \mathbb{L}^{\prime \prime}$ are resolved links in $A \times I$ connected by a saddle cobordism $S$, and $\widehat{\mathbb{L}}^{\prime}$, $\widehat{\mathbb{L}}^{\prime \prime}$ their induced images in $D_{A} \times I$, then the following diagram commutes:

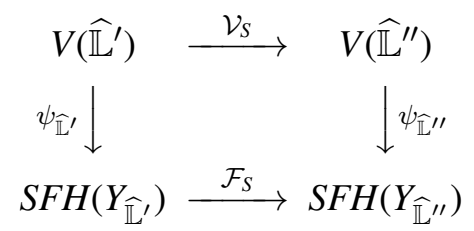

Furthermore, for any resolved $\mathbb{L} \subset A \times I, V(\widehat{\mathbb{L}}) \cong V(\mathbb{L})\left(\right.$ resp., $\left.\operatorname{SFH}\left(Y_{\widehat{\mathbb{L}}}\right) \cong S F H\left(Y_{\mathbb{L}}\right)\right)$ as $\mathbb{Z}_{2}$ vector spaces. In fact, $V(\widehat{\mathbb{L}})$ is obtained from $V(\mathbb{L})$ (resp., $S F H\left(Y_{\widehat{\mathbb{L}}}\right)$ is obtained from $\operatorname{SFH}\left(Y_{\mathbb{L}}\right)$ ) by forgetting the $f$-grading (resp., A-grading), and the isomorphism $\psi_{\widehat{\mathbb{L}}}$ agrees with $\Psi_{\mathbb{L}}$ subject to this forgetful map. Lastly, given any saddle cobordism $S$ connecting a resolved link $\mathbb{L}^{\prime} \subset A \times I$ to $\mathbb{L}^{\prime \prime} \subset A \times I, G \mathcal{V}_{S}$ (resp., $G \mathcal{F}_{S}$ ) is the $f$-grading-preserving (resp., A-grading-preserving) part of the map $\mathcal{V}_{S}$ (resp., $\mathcal{F}_{S}$ ) corresponding to the saddle cobordism connecting $\widehat{\mathbb{L}^{\prime}}$ to $\widehat{\mathbb{L}^{\prime \prime}}$.

Thus, to show that

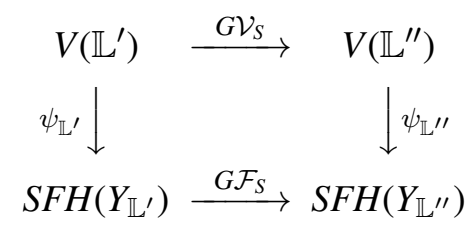


is a commutative diagram it suffices to show that for any two $f$-homogeneous generators $\mathbf{x} \in V\left(\mathbb{L}^{\prime}\right)$ and $\mathbf{y} \in V\left(\mathbb{L}^{\prime \prime}\right)$,

$$
f(\mathbf{x})=f(\mathbf{y}) \Leftrightarrow \mathbf{A}\left(\Psi_{\mathbb{L}^{\prime}}(\mathbf{x})\right)=\mathbf{A}\left(\Psi_{\mathbb{L}^{\prime \prime}}(\mathbf{y})\right) .
$$

But Definitions 2.14 and 2.27 imply that

$$
\begin{aligned}
& \mathbf{A}\left(\Psi_{\mathbb{L}^{\prime}}(\mathbf{x})\right)=\frac{f(\mathbf{x})-p\left(\mathbb{L}^{\prime}\right)}{2}, \text { and } \\
& \mathbf{A}\left(\Psi_{\mathbb{L}^{\prime \prime}}(\mathbf{y})\right)=\frac{f(\mathbf{y})-p\left(\mathbb{L}^{\prime \prime}\right)}{2},
\end{aligned}
$$

so $f$ gradings agree iff the corresponding $\mathbf{A}$ gradings agree. The result follows.

We are now ready to prove Theorem 2.1 .

Proof of Theorem 2.1 We proceed exactly as in the proof of [6, Thm. 5.19]. Recall (see Definition 2.22 and Remark 2.23) that if $\mathbb{L} \subset A \times I$ is a link and $\overline{\mathbb{L}}$ is its mirror, then $K h^{*}(\overline{\mathbb{L}}):=V(\mathbb{L})$, where $V(\mathbb{L})$ is the homology of the cubical complex constructed by

(1) numbering the crossings of a projection, $\mathcal{P}(\mathbb{L})$, of $\mathbb{L}$,

(2) forming the associated cube of resolutions whose vertices are the resolved links, $\mathcal{P}_{\mathcal{I}}(\mathbb{L})$, described in Section 2.2, arranged so that two vertices $\mathcal{I}, \mathcal{I}^{\prime}$ are connected by an edge iff $\mathcal{P}_{\mathcal{I}}(\mathbb{L}), \mathcal{P}_{\mathcal{I}}^{\prime}(\mathbb{L})$ are connected by a saddle cobordism, $S$,

(3) assigning to each vertex of the cube the vector space $V\left(\mathcal{P}_{\mathcal{I}}(\mathbb{L})\right)$ and to each edge of the cube the differential map $G \mathcal{V}_{S}$.

Similarly, using the link surgeries spectral sequence for sutured Floer homology (see [6, Sec. 4]) associated to the link in $Y_{\mathbb{L}}$ formed by taking the preimage of simple arcs at each crossing of the projection $\mathcal{P}(\mathbb{L})$ as in [6, Fig. 7], we see that $\operatorname{SFH}\left(Y_{\mathbb{L}}\right)$ is the homology of the filtered cubical complex constructed by proceeding as in steps (1) and (2) above, but in step (3) assigning the vector space

$$
\operatorname{SFH}\left(Y_{\mathcal{P}_{\mathcal{I}}(\mathbb{L})}\right) \cong V_{H}\left(\mathcal{P}_{\mathcal{I}}(\mathbb{L})\right)
$$

to each vertex of the cube, assigning the map $G \mathcal{F}_{S}$ to each edge of the cube, and assigning higher polygon maps as in [6, Sec. 4] to higher-dimensional faces of the cube.

The $E^{2}$ page of this link surgeries spectral sequence is the homology of the chain complex whose underlying vector space is

$$
\bigoplus_{\mathcal{I} \in\{0,1\}^{\ell}} \operatorname{SFH}\left(Y_{\mathcal{P}_{\mathcal{I}}(\mathbb{L})}\right)
$$


and whose differential consists solely of those maps assigned to the edges of the cube. Proposition 2.29 yields a canonical isomorphism between this chain complex and the chain complex whose homology is $V(\mathbb{L}) \cong K h^{*}(\overline{\mathbb{L}})$. The result follows.

\section{A relationship between annular links and balanced tangles}

We close by establishing the relationship between Roberts' spectral sequence, [28, Prop. 1.1], for annular links (or, more precisely, its reinterpretation in Theorem 2.1) and the spectral sequence for balanced tangles introduced in [6, Prop. 5.20]. The following is a direct consequence of [7, Thm. 4.5], which generalizes Juhász's surface decomposition theorem, [9, Thm. 1.3], to filtered complexes arising in the context of link surgeries spectral sequences. See [7] for a more complete discussion of related naturality results.

Theorem 3.1 Let $\mathbb{L} \subset A \times I$ be an isotopy class representative of an annular link admitting a projection, $\mathcal{P}(\mathbb{L})$, and let $\lambda \subset A$ be a properly imbedded oriented arc representing a nontrivial element of $H_{1}(A, \partial A)$ such that $\lambda$ intersects $\mathcal{P}(\mathbb{L})$ transversely. Let $T \subset D \times I$ be the balanced tangle in $D \times I$ obtained by decomposing along the surface, $\lambda \times I$, endowed with the product orientation.

Then the spectral sequence

$$
K h^{*}(\bar{T}) \rightarrow \operatorname{SFH}(\Sigma(D \times I, T))
$$

is a direct summand of the spectral sequence

$$
K h^{*}(\overline{\mathbb{L}}) \rightarrow \operatorname{SFH}(\Sigma(A \times I, \mathbb{L})) .
$$

Furthermore, the direct summand is trivial if there exists some $\mathbb{L}^{\prime} \subset A \times I$ isotopic to $\mathbb{L}$ satisfying

$$
\left|(\lambda \times I) \pitchfork \mathbb{L}^{\prime}\right| \lesseqgtr|(\lambda \times I) \pitchfork \mathbb{L}|
$$

\section{Proof of Theorem 3.1 Let}

- $\vec{p}:=(\lambda \times I) \pitchfork \mathbb{L}$ denote the collection of transverse intersection points of $\mathbb{L}$ with $\lambda \times I$,

- $S:=\Sigma(\lambda \times I, \vec{p})$ denote the preimage of $\lambda \times I$ in $\Sigma(A \times I, \mathbb{L})$, and

- $L$ denote the link in $\Sigma(A \times I, \mathbb{L})$ obtained as the preimage of trivial arcs at each crossing of $\mathbb{L}$ as in [6, Fig. 7]. 
Then $S, L \subset \Sigma(A \times I, \mathbb{L})$ satisfy the assumptions of [7, Thm. 4.5]. Furthermore, the decomposition of $\Sigma(A \times I, \mathbb{L})$ along $S$ is precisely the sutured manifold $\Sigma(D \times I, T)$, and $L^{\prime} \subset \Sigma(D \times I, T)$, the image of $L$ in the decomposed manifold, is precisely the link in $\Sigma(D \times I, T)$ obtained as the preimage of the trivial arcs associated to the crossings of $\mathcal{P}(T) \subset D$.

Now [7, Thm. 4.5] implies that the filtered complex, $X\left(L^{\prime}\right)$, associated to $L^{\prime}$ is a direct summand of the filtered complex, $X(L)$, associated to $L$. But $X\left(L^{\prime}\right)$ is precisely the filtered complex whose associated spectral sequence has $E^{2}$ term $K h^{*}(\bar{T})$ and converges to $\operatorname{SFH}(\Sigma(D \times I, T))$, while the spectral sequence associated to $X(L)$ has $E^{2}$ term $K h^{*}(\overline{\mathbb{L}})$ and converges to $\operatorname{SFH}(\Sigma(A \times I, \mathbb{L}))$, as desired.

Furthermore, the direct summand, $X\left(L^{\prime}\right) \leq X(L)$, is trivial if the intersection of $\lambda \times I$ with $\mathbb{L}$ is non-minimal, since we know that if $|\mathbb{L} \pitchfork(\lambda \times I)|$ is non-minimal among isotopy class representatives of $\mathbb{L}$, then all resolutions of $T$ backtrack, in the sense of [6, Defn. 5.8], so the associated chain complex for $K h(T)$ has no generators, hence the entire filtered complex, $X\left(L^{\prime}\right)$, and its associated spectral sequence are trivial.

\section{References}

[1] Marta Asaeda, Józef H. Przytycki, and Adam S. Sikora. Categorification of the skein module of tangles. math.QA/0410238, 2004.

[2] Marta M. Asaeda, Józef H. Przytycki, and Adam S. Sikora. Categorification of the Kauffman bracket skein module of I-bundles over surfaces. Algebr. Geom. Topol., 4:1177-1210 (electronic), 2004.

[3] Kenneth L. Baker, J. Elisenda Grigsby, and Matthew Hedden. Grid diagrams for lens spaces and combinatorial knot Floer homology. Int. Math. Res. Notices, doi: 10.1093/imrn/rnn024, 2008.

[4] John Baldwin and Olga Plamenevskaya. Khovanov homology, open books, and tight contact structures. math.GT/0808.2336, 2008.

[5] David Gabai. Foliations and the topology of 3-manifolds. J. Differential Geom., 18(3):445503, 1983.

[6] J. Elisenda Grigsby and Stephan M. Wehrli. On the colored Jones polynomial, sutured Floer homology, and knot Floer homology. Advances in Mathematics, 223:2114-2165, 2009.

[7] J. Elisenda Grigsby and Stephan M. Wehrli. On the naturality of the spectral sequence from Khovanov homology to Heegaard Floer homology. Int. Math. Res. Notices, doi: 10.1093/imrn/rnq039, 2010.

[8] András Juhász. Holomorphic discs and sutured manifolds. Algebr. Geom. Topol., 6:14291457 (electronic), 2006. 
[9] András Juhász. Floer homology and surface decompositions. Geom. Topol., 12(1):299-350, 2008.

[10] Mikhail Khovanov. A categorification of the Jones polynomial. Duke Math. J., 101(3):359426, 2000.

[11] Mikhail Khovanov. Patterns in knot cohomology. I. Experiment. Math., 12(3):365-374, 2003.

[12] Mikhail Khovanov. Categorifications of the colored Jones polynomial. J. Knot Theory Ramifications, 14(1):111-130, 2005.

[13] Ciprian Manolescu, Peter Ozsváth, and Sucharit Sarkar. A combinatorial description of knot Floer homology. math.GT/0607691, 2006.

[14] Ciprian Manolescu, Peter Ozsváth, Zoltán Szabó, and Dylan Thurston. On combinatorial link Floer homology. math.GT/0610559, 2006.

[15] Yi Ni. Link Floer homology detects the Thurston norm. math.GT/0604360, 2006.

[16] Peter Ozsváth and Zoltán Szabó. Knot Floer homology and the four-ball genus. Geom. Topol., 7:615-639 (electronic), 2003.

[17] Peter Ozsváth and Zoltán Szabó. Holomorphic disks and knot invariants. Adv. Math., 186(1):58-116, 2004.

[18] Peter Ozsváth and Zoltán Szabó. Holomorphic disks and three-manifold invariants: properties and applications. Ann. of Math. (2), 159(3):1159-1245, 2004.

[19] Peter Ozsváth and Zoltán Szabó. Holomorphic disks and topological invariants for closed three-manifolds. Ann. of Math. (2), 159(3):1027-1158, 2004.

[20] Peter Ozsváth and Zoltán Szabó. Heegaard Floer homology and contact structures. Duke Math. J., 129(1):39-61, 2005.

[21] Peter Ozsváth and Zoltán Szabó. Holomorphic disks and link invariants. math.GT/0512286, 2005.

[22] Peter Ozsváth and Zoltán Szabó. On knot Floer homology and lens space surgeries. Topology, 44(6):1281-1300, 2005.

[23] Peter Ozsváth and Zoltán Szabó. On the Heegaard Floer homology of branched doublecovers. Adv. Math., 194(1):1-33, 2005.

[24] Peter Ozsváth and Zoltán Szabó. Holomorphic triangles and invariants for smooth fourmanifolds. Adv. Math., 202(2):326-400, 2006.

[25] Olga Plamenevskaya. Transverse knots and Khovanov homology. math.GT/0412184, 2004.

[26] Jacob A. Rasmussen. Khovanov homology and the slice genus. math.GT/0402131, 2004.

[27] Jacob Andrew Rasmussen. Floer homology and knot complements. PhD thesis, Harvard University, 2003.

[28] Lawrence P. Roberts. On knot Floer homology in double branched covers. math.GT/0706.0741, 2007. 
[29] Liam Watson. Surgery obstructions from Khovanov homology. math.GT/0807.1341, 2008.

Boston College Mathematics Department, 301 Carney Hall, Chestnut Hill, MA 02467

Syracuse University Mathematics Department, 215 Carnegie, Syracuse, NY 13244

grigsbyj@bc.edu, smwehrli@syr.edu 